\title{
Violazione delle garanzie "processuali" della CEDU e rimedi post-iudicatum
}

\author{
Violation of ECHR "procedural" \\ guarantees and post-iudicatum remedies
}

Violações das garantias "processuais"
da CEDH e remédios post-iudicatum

Barbara Lavarini ${ }^{1}$

Università degli Studi di Torino/Italia

barbara.lavarini@unito.it

http://lattes.cnpq.br/1895055427947442

https://orcid.org/0000-0003-1227-9101

\begin{abstract}
RIASSUNTO: Lo scritto analizza i rimedi disponibili in Italia per dare esecuzione alle sentenze della Corte europea dei diritti umani che, nel condannare lo Stato per la violazione di garanzie convenzionali "processuali", individuino nella riapertura post iudicatum del processo un idoneo strumento di ristoro della vittima della violazione. Nella perdurante inerzia del legislatore, è stata la Corte costituzionale a introdurre la c.d. revisione "europea", innestandola però su un istituto - la revisione "tradizionale" - dagli obiettivi affatto diversi, la cui disciplina, quindi, è per molti versi inadatta a recepire il nuovo rimedio. Di qui la difficoltà di individuare presupposti, modalità di svolgimento ed esiti decisori della revisione "europea", conciliando gli ampi margini di discrezionalità interpretativa riconosciuti dalla Corte costituzionale al giudice ordinario col principio di legalità processuale. Al rimedio introdotto dal Giudice delle leggi si è peraltro recentemente affiancato il nuovo istituto della rescissione del giudicato, che, già ora fruibile a ristoro delle garanzie convenzionali del processo in absentia, potrebbe in futuro offrire il
\end{abstract}

1 Professoressa associata di Diritto processuale penale - Università degli Studi di Torino. 
modello a cui ricondurre, con qualche adattamento, ogni ipotesi di riapertura del processo in adeguamento ai canoni convenzionali.

Parole chiave: giudicato; Corte europea dei diritti dell'uomo; riapertura del processo; revisione.

ABSTRACT: The paper analyzes the remedies available in Italy to implement judgments of the European Court of Human Rights which identitify the retrial as a suitable means to ensure that the injured party is restored in a conventional "trial" guarantee. Due to Italian legislator's inertia, the Constitutional Court introduced the "European" review, using as a model a completely different institute - the "traditional" review - whose regulation is, therefore, in many respects unsuitable to implement the new remedy. Thus, it is quite hard to identify the conditions, procedures and results of the "European" review. It is also hard to reconcile the wide interpretative discretion granted by the Constitutional Court on judges with the principle of procedural legality. Moreover, a new institute - the "rescissione" of res iudicata - was recently added to the review. Such remedy would already be suitable to restore the conventional guarantees of the process in absentia, but in future it could be taken as a model for any kind of retrial in compliance with conventional guarantees.

KEY wORDS: res iudicata; European Court of Human rights; review; retrial.

Resumo: O texto analisa os remédios disponíveis na Itália para dar execução às sentenças do Tribunal Europeu dos Direitos Humanos que, condenando o Estado pela violação das garantias convencionais "processuais", determinam a reabertura post iudicatum do processo como um idôneo instrumento de reparação às vítimas das violações. Na contínua inércia do legislador, foi a Corte Constitucional quem introduziu a denominada "revisão europeia", enxertando-a, todavia, sobre um instituto - a revisão tradicional - cujas finalidades são profundamente diferentes, e cuja disciplina, portanto, aparece inadequada a receber o novo remédio processual. Neste contexto delineado, verifica-se a dificuldade de especificar pressupostos, modalidades de desenvolvimento e êxitos decisórios da revisão "europeia", conciliando as amplas margens de discricionariedade interpretativas reconhecidas pela Corte Constitucional italiana em favor do juiz ordinário em relação ao princípio de legalidade processual. Ademais, ao remédio introduzido pelo "Juiz das Leis" foi recentemente adicionado o novo instituto da rescisão do trânsito em julgado, que atualmente é utilizável como restauração das garantias convencionais do processo in absentia, $e$ poderia futuramente oferecer o modelo ao qual reconduzir, com algumas 
adequações, qualquer hipótese de reabertura do processo em conformação aos cânones convencionais.

Palavras-chave: coisa julgada; Tribunal Europeu dos Direitos Humanos; reabertura do processo; revisão.

Sommario: 1. Premessa. 2. Dai rimedi "pretori" alla revisione "europea". 3. L'ambito applicativo del nuovo caso di revisione: a) premessa metodologica. 4. b) L'oggetto e i soggetti dell'impugnazione straordinaria. 5. c) I presupposti. 6. II giudizio di revisione e i relativi esiti: la "traduzione" delle violazioni convenzionali nei vizi processuali interni. 7. I confini tra la revisione "europea" e la rescissione del giudicato. 8. Spunti de iure condendo.

\section{Premessa}

Ormai da tempo il principio di intangibilità del giudicato penale è oggetto di un inarrestabile "processo erosivo"2 per via dei riflessi, nell'ordinamento interno, della normativa e della giurisprudenza internazionali e sovranazionali, che, nell'intento di assicurare una più ampia e uniforme tutela dei diritti umani, si stanno dimostrando sempre più "pervasive" anche in settori - come appunto la matière pénale - tradizionalmente lasciati all'autonomia dei singoli Stati.

Il riferimento va, in particolare, all'obbligo, per ciascuno Stato membro del Consiglio d'Europa, di «conformarsi» - cioè di dare esecuzione - alle sentenze definitive della Corte europea dei diritti umani che riscontrino una violazione, ad opera di quello Stato, delle garanzie convenzionali (art. $46 \mathrm{CEDU})^{3}$. Considerato, infatti, che la Corte di Strasburgo può essere adita solo «previo esaurimento delle vie di ricorso interne» (art. 35 CEDU), va da sé che l'adozione delle misure individuali o generali,

2 Fra gli altri, SCIARABBA, Vincenzo. Il giudicato e la Cedu. Padova: Cedam, 2012, p. XIV.

3 Per un inquadramento generale, KOSTORIS, Roberto. Diritto europeo e giustizia penale. In: KOSTORIS, Roberto (a cura di.). Manuale di procedura penale europea. Torino: Giappichelli, $3^{\text {a }}$ ed., 2017, p. 62 ss. 
diverse da una mera riparazione pecuniaria, prescritte dalla Corte stessa a ristoro dell'accertata violazione ${ }^{4}$, si pone spesso fisiologicamente a valle del giudicato, imponendone - secondo i casi -, la cessazione degli effetti, la modifica o la rimozione ${ }^{5}$.

L'esigenza, per gli Stati membri, di dotarsi di strumenti idonei ad un tale obiettivo, emergeva già dalla Raccomandazione n. R (2000) 2 del Comitato dei ministri del Consiglio d'Europa ${ }^{6}$, ove si rilevava come, seppure spetti ai singoli Stati “condannati”, secondo le peculiarità dei rispettivi ordinamenti, «decidere quali misure siano più appropriate per assicurare una restitutio in integrum», in ipotesi eccezionali - in primis allorché la vittima della violazione convenzionale continui a subirne, per via del giudicato interno, effetti gravemente pregiudizievoli - il «mezzo più efficace, se non addirittura l'unico», funzionale allo scopo, consista nel «riesame di un caso» o nella «riapertura del procedimento». Ciò a seconda, rispettivamente, che la decisione interna, alla base del ricorso alla Corte europea, sia stata ritenuta nel merito contraria alla Convenzione - come accade a fronte del riscontro della violazione di una garanzia

4 La Corte europea ha da tempo abbandonato l'originario self-restraint che la induceva ad un pressoché esclusivo ricorso, a ristoro della violazione convenzionale riscontrata, ad una riparazione pecuniaria; ciò in piena conformità, del resto, all'art. 41 CEDU, che individua nella restitutio in integrum l'effetto primario dell'accertamento della violazione, riservando la riparazione pecuniaria ai casi, residuali, in cui «il diritto interno dell'Alta Parte contraente non permetta che in modo incompleto di riparare le conseguenze» della violazione stessa. Di qui una sempre più frequente indicazione - talora nel dispositivo, più spesso nella motivazione del provvedimento - delle misure individuali o generali necessarie o comunque appropriate al ristoro della garanzia. In dottrina, per una sintetica ricostruzione del percorso seguito dalla giurisprudenza europea, cfr. PIRRONE, Pasquale. Sub art. 46. In: BARTOLE, Sergio; DE SENA, Pasquale; ZAGREBELSKY, Vladimiro (a cura di). Commentario breve alla CEDU. Padova: Cedam, 2012, p. 746

5 Fra gli altri, CALLARI, Francesco. La revisione. La giustizia penale tra forma e sostanza. Torino: Giappichelli, 2012, p. 274; IACOVIELLO, Francesco Maria. Il quarto grado di giurisdizione: la Corte europea dei diritti dell'uomo. Cassazione penale, 2011, p. 816; MAZZA, Oliviero. La procedura penale. In VIGANÒ, Francesco; MAZZA, Oliviero. Europa e giustizia penale. Diritto penale e processo. Speciali, 2011, p. 47.

6 Organo deputato, a norma dell'art. 46 §§ 2-5 CEDU, a «sorveglia[re] l'esecuzione» delle sentenze della Corte europea. 
"sostanziale" -, o che si siano riscontrate violazioni "processuali" ${ }^{8}$ di tale gravità da determinare seri dubbi sull'esito del procedimento nazionale» ${ }^{9}$. Successivamente, la linea tracciata dalla Raccomandazione è stata ribadita - assumendo così un più autorevole "crisma" giurisdizionale - da una copiosa giurisprudenza della Corte di Strasburgo, e da ultimo dalla Grande Camera ${ }^{10}$, la quale, sia pure in termini molto "guardinghi" - ciò che ha determinato un elevato numero di opinioni dissenzienti ${ }^{11}-$, ha ricordato come «in eccezionali circostanze», più facilmente riscontrabili in relazione ai procedimenti penali, «il riesame del caso o la riapertura del processo si dimostri il più efficiente, se non l'unico, strumento di restitutio in integrum in favore della vittima della violazione».

Nella pressoché totale inerzia del legislatore - intervenuto ripetutamente, sulla scia della giurisprudenza della Corte dei diritti umani, nel solo settore del procedimento in absentia ${ }^{12}$-, la "via italiana" all'individuazione di un rimedio post iudicatum in adeguamento ai canoni

7 Si pensi alle garanzie ex artt. 7 (principio di legalità penale), 9 (libertà di pensiero, coscienza, religione), 10 (libertà di espressione) CEDU. Per alcuni esempi cfr. l'Explanatory memorandum Rec. No. R (2000) 2, disponibile in https:// search.coe.int/cm/Pages/result_details.aspx?ObjectID=09000016805e2f06. Accesso: 13 agosto 2018, ove si includono tra le violazioni "sostanziali" «criminal convictions violating article 10 because the statements characterised as criminal by the national authority constitute legitimate exercise of the injuried party's freedom of expression, or violating art. 9 because the behaviour characterised as criminal is a legitimate exercise of freedom of religion».

8 Fra gli esempi proposti nell'Explanatory memorandum, cit., si rinviene il caso in cui «the injuried party did not have the time and facilities to prepare his or her defence in criminal proceedings», oppure «the conviction was based ...on material which the injuried party had no possibility of verifying».

9 Cfr. Racc. n. $R$ (2000) 2 del 19 gennaio 2000, concernente il riesame o la riapertura di certi casi nell'ordinamento nazionale a seguito delle sentenze della Corte europea dei diritti dell'uomo (la traduzione italiana è reperibile in Diritto penale e processo, 2000, p. 391).

10 Corte eur., Gr. Camera, 11 luglio 2017, Moreira-Ferreira c. Portogallo, §§ 4852, in <https://hudoc.echr.coe.int>. Accesso: 13 agosto 2018

11 Si veda in particolare l'articolata dissenting opinion del giudice Paulo Pinto de Albuquerque - a cui hanno aderito i giudici Karakas, Sajo, Lazarova Trjkowska, Tsotsoria, Vehabovic e Kuris - volta a lamentare l'eccessiva timidezza della Grande Camera nel delineare i contorni dell'obbligo, ex art. 46 CEDU, di riaprire il procedimento interno "affetto" da violazioni convenzionali.

12 V. meglio, infra, § 7. 
convenzionali si è rivelata assai tortuosa, passando prima per discutibili soluzioni "pretorie", poi per il "generoso" intervento additivo con cui la Corte costituzionale ha introdotto la c.d. revisione "europea"13, peraltro inidonea ad offrire copertura esaustiva, a fronte dell'eterogeneità delle violazioni convenzionali e delle relative prescrizioni riparatorie. Né le recenti riforme, che pure hanno interessato ampi settori della procedura penale - ivi compresa la disciplina delle impugnazioni, anche straordinarie ${ }^{14}$ - sono state sfruttate dal legislatore per "mettere ordine" nella materia che ci occupa, tuttora affidata in gran parte all'elaborazione giurisprudenziale, in contrasto coi canoni di stretta legalità processuale imposti dall'art. 111 comma 1 Cost. ${ }^{15}$

In un siffatto contesto, scopo del presente lavoro è la ricostruzione dei presupposti, dell'ambito applicativo e delle difficoltà operative della revisione "europea" e di alcuni strumenti "limitrofi" di caducazione del giudicato conseguente a un processo "convenzionalmente illegittimo". Ciò nell'intento non soltanto di illustrare lo "stato dell'arte", ma anche di trarne qualche spunto de iure condendo.

\section{Dal Rimedi "pretori" alla revisione "europea"}

A fronte delle prime sentenze con cui la Corte dei diritti umani, nel condannare l'Italia per la violazione, in danno del ricorrente, delle garanzie del fair trial, ha specificamente sollecitato il ripristino dello status anteriore alla violazione - sino alla riapertura del processo per consentirne la celebrazione "convenzionalmente compatibile" -, è stata la giurisprudenza ordinaria a cercare gli strumenti attuativi dei dicta di Strasburgo, piegando alle nuove necessità istituti interni nati per altro scopo, quali l'incidente di esecuzione ex artt. 666 e 670 c.p.p. - in grado,

13 Corte cost. 7 aprile 2011, n. 113, Giurisprudenza costituzionale, 2011, p. 1523.

14 Il riferimento è in particolare alla 1.23 giugno 2017, n. 103, che pur rimodulando l'istituto della rescissione del giudicato formatosi in absentia, ha perso l'occasione per innestare su tale istituto un rimedio di portata generale alle violazioni "europee" (v. meglio, infra, § 8).

15 Sul principio di legalità processuale v., recentemente, NEGRI, Daniele. Splendori e miserie della legalità processuale. Archivio penale, 2017, n. 2, p. 421 ss. https://doi.org/10.12871/9788674101944 
peraltro, di offrire soluzioni soltanto parziali ${ }^{16}-$, e il ricorso straordinario per cassazione ex art. 625-bis c.p.p., discutibilmente adattato "per analogia” alle esigenze europee ${ }^{17}$, in contrasto col principio di tassatività delle impugnazioni, a maggior ragione se straordinarie ${ }^{18}$.

La "via maestra" verso un rimedio di più ampia portata veniva successivamente individuata - anche prendendo spunto da una serie di

16 Il rimedio in oggetto, utilizzato la prima volta da Cass., Sez. I, 1 dicembre 2006, Dorigo, Cassazione penale, 2007, p. 1441 - in esecuzione di una decisione europea che aveva ravvisato la violazione del diritto del ricorrente di interrogare i testimoni a carico ex art. $6, \S 3$, lett. d) CEDU - garantiva, infatti, la cessazione di un'esecuzione illegale - sul presupposto che, data la correlazione fra gli artt. 6 e 5 lett. a) CEDU, non possa dirsi «regolare», agli effetti di quest'ultima disposizione, la detenzione fondata su una condanna seguente a un processo ingiusto -, ma non la riapertura del procedimento, così determinandosi «la paradossale situazione di un giudicato penale non più eseguibile ma non ancora rinnovabile...collocato in una sorta di limbo processuale»: così UBERTIS, Giulio. Corte europea dei diritti dell'uomo e processo equo: riflessi sul processo penale italiano. Rivista di diritto processuale, 2009, p. $43 \mathrm{~s}$.

17 Nel dettaglio, il rimedio veniva utilizzato da Cass., Sez. VI, 12 novembre 2008, Drassich, Cassazione penale, 2009, p. 1457 onde riaprire il giudizio di cassazione in esecuzione di Corte eur., Sez. II, 11 dicembre 2007, Drassich c. Italia (in <https://hudoc.echr.coe.int>. Accesso 13 agosto 2018), che aveva ritenuto in contrasto con l'art. $6, \S 3$, lett. a) e b) CEDU, la riqualificazione in peius del fatto operata dalla Corte di legittimità nel rigettare il ricorso per cassazione, con conseguente impossibilità, per l'imputato, di esercitare il contraddittorio sul nuovo nomen iuris. Secondo la Cassazione il ricorso straordinario, seppure esperibile al solo fine di riparare gli errori materiali o di fatto incorsi nel giudizio di legittimità (art. 625-bis comma 1 c.p.p.), risultava fruibile nel caso di specie «per analogia», sfuggendo al divieto ex art. 14 disp. prel. c.c. in quanto «non si caratterizza per eccezionalità rispetto al sistema processuale», nel quale, al contrario, ha colmato «un vuoto normativo dovuto all'inadeguatezza della precedente disciplina a tutelare anomalie e violazioni riconducibili al diritto di difesa, pur configurabili con ordinarietà nel giudizio di legittimità». Di qui, l'identità di ratio fra il rimedio de quo e quello sollecitato dal giudicato europeo, l'uno e l'altro funzionali a riparare «oltre che...veri e propri errori di fatto...violazioni del diritto di difesa occorse nell'ambito del giudizio di legittimità», che abbiano reso «invalida per iniquità» la sentenza di cassazione.

18 Fra gli altri, AIMONETTO, Maria Gabriella. Condanna "europea” e soluzioni interne al sistema processuale penale: alcune riflessioni e spunti de iure condendo. Rivista italiana di diritto e procedura penale, 2009, p. 1512; ZACCHÉ, Francesco. Cassazione e iura novit curia nel caso Drassich. Diritto penale e processo, 2009, p. 784. 
iniziative legislative poi arenatesi in Parlamento ${ }^{19}$ - in un adeguamento della disciplina della revisione, che, giustamente reputato impossibile per via interpretativa, veniva sollecitato al Giudice delle leggi.

Dopo che una prima questione di costituzionalità fu dichiarata infondata ${ }^{20}$, una seconda questione, più correttamente sollevata in relazione agli artt. 117 Cost. e 46 CEDU, ha infine condotto all'introduzione della revisione "europea", attraverso la dichiarazione di illegittimità costituzionale dell'art. 630 c.p.p. laddove non prevede «un diverso caso di revisione della sentenza o del decreto penale di condanna al fine di conseguire la riapertura del processo, quando ciò sia necessario, ai sensi dell'art. 46 par. 1 CEDU, per conformarsi ad una sentenza della Corte europea dei diritti dell'uomo $»^{21}$.

Tale intervento additivo era, in realtà, tutt'altro che scontato. Come da più parti osservato, infatti, il rimedio post iudicatum, necessario a garantire la riapertura dei procedimenti di accertata "illegalità convenzionale", presenta tratti radicalmente eterogenei rispetto al tradizionale ambito della revisione, a tacer d'altro perché questa è strettamente funzionale al proscioglimento - tanto che la "prognosi assolutoria" ne costituisce condizione di ammissibilità -, mentre la rinnovazione del procedimento - o di alcuni suoi atti - secondo i canoni del fair trial ben potrebbe condurre a ribadire il giudicato di condanna ${ }^{22}$. Ciononostante il Giudice delle leggi, posto «di fronte ad un vulnus costituzionale non sanabile per via interpretativa», e preso atto dell'insensibilità del legislatore al problema, ha ritenuto che la revisione, siccome idonea a garantire la riapertura del processo anche agli effetti dell'assunzione probatoria, fosse l'istituto «di maggiore assonanza» - fra i rimedi post iudicatum of-

19 Per un quadro di sintesi GERACI, Rosa Maria. Sentenze della Corte E.D.U. e revisione del processo penale. Roma: Dike, 2012, p. 84 ss.

20 Cfr. Corte cost. 30 aprile 2008, n. 129, Giurisprudenza costituzionale, 2008, p. 1506.

21 Corte cost., 7 aprile 2011, n. 113, cit.

22 Fra gli altri AIMONETTO, Maria Gabriella. Condanna “europea”, cit., p. 1512; MANTOVANI, Giulia. La sentenza n. 129 del 2008 e la riparazione delle violazioni dell'art. 6 CEDU. Giurisprudenza costituzionale, 2008, p. 2680 s.; MAZZA, Oliviero. L'esecuzione può attendere: il caso Dorigo e la condanna ineseguibile per accertata violazione della CEDU. Giurisprudenza italiana, 2007, p. 2640. 
ferti dal sistema allora vigente - «con quello la cui introduzione appare necessaria a garantire la conformità dell'ordinamento nazionale» agli artt. 117 Cost. e 46 CEDU $^{23}$.

Né la Corte costituzionale ha ravvisato un ostacolo nella carenza di disciplina in cui l'inadattabilità, su più fronti, del regime della revisione "ordinaria" (artt. 629 ss. c.p.p.), finiva per abbandonare il nuovo istituto: secondo il Giudice delle leggi, spetta infatti al giudice comune - nell'attesa di un pur sempre auspicabile intervento legislativo - «il vaglio di compatibilità delle singole disposizioni relative al giudizio di revisione» col rimedio introdotto per via additiva, nel rispetto di alcuni "punti fermi" indicati dalla stessa Corte.

Col che la giurisprudenza costituzionale - come già quella ordinaria - si è dimostrata propensa a sacrificare "sull'altare" dei vincoli internazionali - sia pure in nome di una più ampia tutela dei diritti principi fondamentali interni di primario rilievo, a partire da quello di riserva di legge in materia processuale (art. 111 comma 1 Cost.), in cui la più attenta dottrina aveva invece letto, condivisibilmente, un limite anche all'eccessivo interventismo della Corte costituzionale ${ }^{24}$.

E' soltanto il caso di accennare - dato il fulcro "penalistico" del presente lavoro - che il Giudice delle leggi ha mantenuto un atteggiamento assai più cauto allorché si è trovato a decidere della riapertura - in adeguamento a una sentenza della Corte di Strasburgo - di un giudizio civile o amministrativo. Nel dichiarare infondate due questioni, relative alla revocazione del giudicato civile (art. 395 c.p.c.), apparentemente speculari a quella risolta positivamente dalla sentenza n. 113 del 2011, la Corte costituzionale ha infatti affermato che, quando non sia in gioco la libertà personale, la delicata ponderazione degli interessi coinvolti da

23 In ragione della sua particolarità, la sentenza n. 113 del 2011 è stata efficacemente qualificata «additiva di istituto»: GIALUZ, Mitja. Una sentenza "additiva di istituto": la Corte costituzionale crea la "revisione europea". Cassazione penale, 2011, p. 3308 ss.

24 FERRUA, Paolo. Il 'giusto processo'. Bologna: Zanichelli, $3^{\text {a }}$ ed., 2012, p. 98 s. Con specifico riguardo al tema che ci occupa, cfr., per il richiamo al principio di riserva di legge in materia processuale, GIALUZ, Mitja. Una sentenza "additiva di istituto", cit., p. 3311; NEGRI, Daniele. I confini della legalità processuale. Diritto penale e processo, 2007, p. 1229 ss. 
un'eventuale revoca del giudicato - potenzialmente lesiva delle parti del giudizio a quo non ricorrenti a Strasburgo - spetta in via prioritaria al legislatore, non prestandosi a un intervento additivo ${ }^{25}$. Del resto, la stessa giurisprudenza convenzionale riconosce la significativa differenza quando si tratti di intervenire sul giudicato in adempimento dei precetti europei - fra procedimenti civili e penali, anche in ragione della tutela delle parti "terze" rispetto al giudizio celebratosi a Strasburgo ${ }^{26}$.

\section{L'AMBITO APPLICATIVO DEL NUOVO CASO DI REVISIONE: A) PREMESSA METODOLOGICA}

Come abbiamo anticipato, talune indicazioni di base, funzionali a delimitare l'ambito del nuovo caso di revisione, si ricavano direttamente dalla sentenza costituzionale che ne ha segnato l'introduzione, mentre la disciplina "di dettaglio" è stata - discutibilmente - demandata al giudice ordinario, e dovrà quindi evincersi per via interpretativa dalla disciplina della revisione "tradizionale", con un'operazione di "adattamento" alle esigenze europee.

Sotto quest'ultimo profilo, una premessa metodologica appare opportuna. Giusta il principio di legalità processuale, infatti, può al più tollerarsi un adattamento per sottrazione, che escluda la riferibilità alla revisione "speciale" delle disposizioni ex artt. 629 ss. con essa inconci$\operatorname{liabili}^{27}$. Va invece radicalmente escluso, a nostro parere, un adattamento per addizione, che introduca per via giurisprudenziale profili di disciplina privi di ogni aggancio nella revisione "tradizionale". Una circoscritta eccezione a questo criterio potrà ammettersi, al più, quanto alle "regole" direttamente enunciate dalla stessa sentenza n. 113 del $2011^{28}$.

25 Corte cost., 26 maggio 2017, n. 123, Giurisprudenza costituzionale, 2017, p. 1246; Id., 27 aprile 2018, n. 93, in <http://www.giurcost.org>. Accesso: 13 agosto 2018.

26 Corte eur., Gr. Camera, 11 luglio 2017, Moreira-Ferreira c. Portogallo, cit., §§ 66 e 67.

27 Sia pure in una prospettiva parzialmente diversa da quella qui coltivata, allude ad un'operazione di adattamento "per sottrazione" SCIARABBA, Vincenzo. Il giudicato e la Cedu, cit., p. 70.

28 Cfr. SCIARABBA, Vincenzo. Il giudicato e la Cedu, cit., p. 68. 


\section{B) L'OGgETTO E I SOGGETTI DELL'IMPUGNAZIONE STRAORDINARIA}

Va in primo luogo sottolineato che, data la declaratoria di incostituzionalità dell'art. 630 c.p.p. in quanto non prevede un diverso caso di revisione «della sentenza o del decreto penale di condanna», il rimedio appare esperibile contro questi soli provvedimenti, con l'aggiunta della sentenza applicativa della pena su richiesta delle parti, giusta, da un lato, l'equiparazione alla sentenza di condanna (art. 445 c.p.p.), dall'altro, l'espressa inclusione fra i provvedimenti suscettibili di revisione "tradizionale” (art. 629 c.p.p. come modificato dalla l. 12 giugno 2003, n. 134) ${ }^{29}$.

Non sembra quindi "coperta" dal dispositivo della sentenza costituzionale alcuna tipologia di proscioglimento, finanche altamente pregiudizievole per il prosciolto (si pensi alla sentenza di assoluzione per difetto di imputabilità con cui si disponga l'applicazione di una misura di sicurezza).

Deve però darsi atto che in materia di revisione "tradizionale" anch'essa circoscritta, ex art. 629 c.p.p., ai provvedimenti di condanna ${ }^{30}-$ la Cassazione ha recentemente sostenuto che proprio la giurisprudenza convenzionale, attraverso la nozione autonoma di "sanzione penale", imporrebbe di qualificare 'condanna' - agli effetti del rimedio post-iudicatum - «ogni provvedimento con il quale il giudice, al di là del nomen iuris, nella sostanza infligga una sanzione che abbia comunque natura punitiva e deterrente, e non meramente riparatoria o preventiva» ${ }^{31}$. Un siffatto ampliamento dell'ambito applicativo del rimedio certo risponderebbe alla ratio - di conformazione all'art. 46 CEDU - che ha indotto la Corte costituzionale a introdurre la revisione "europea". Del resto, lo stesso Giudice delle leggi - sia pure per via meramente interpretativa - ha talora accolto una nozione "sostanziale" di condanna per giustificare l'applicazione di misure "convenzionalmente penali” ad opera di una sentenza di proscioglimento, rilevando come non

29 V. anche LORENZETTO, Elisa. Violazioni convenzionali e tutela post iudicatum dei diritti umani, in <http://www.lalegislazionepenale.eu>, 25 novembre 2016, p. 11. Accesso: 13 agosto 2018.

30 L'esclusione dall'ambito di applicabilità del rimedio del proscioglimento "pregiudizievole" è criticata in dottrina: fra gli altri, SCALFATI, Adolfo. L'esame sul merito nel giudizio preliminare di revisione. Padova: Cedam, 1995, p. 46.

31 Cass., Sez. II, 25 ottobre 2017, n. 53678, Ricupati, in <http://www.italgiure. giustizia.it/sncass/>. Accesso 13 agosto 2018. 
conti - a tal fine - la qualifica formale, bensì l'accertamento di responsabilità eventualmente sotteso, nel caso concreto, all'esito proscioglitivo ${ }^{32}$. E questa lettura "sostanziale" ha da ultimo ricevuto l'espresso avallo della Grande Camera della Corte di Strasburgo proprio in una vicenda italiana, ove si discuteva della compatibilità con l'art. 7 CEDU della confisca "urbanistica" (art. 44 D.P.R. 6 giugno 2001, n. 380) disposta con sentenza di proscioglimento per prescrizione del reato ${ }^{33}$.

Va da sé che, accolta in damnosis una siffatta nozione estesa di condanna, parrebbe ragionevole applicarla altresì per garantire un rimedio al "prosciolto", nel caso in cui la Corte europea lo riconosca vittima di una violazione convenzionale.

Dubitiamo però che il risultato - frutto dell'analogia, sia pure in una prospettiva "convenzionalmente orientata" - possa essere conseguito per via interpretativa, dato l'ostacolo opposto dal principio di "tassatività al quadrato" delle impugnazioni straordinarie. Piuttosto, si dovrebbe investire la Corte costituzionale della questione di legittimità della norma - frutto del suo stesso intervento additivo - che esclude dall'ambito della revisione i proscioglimenti più gravemente pregiudizievoli, a fronte della relativa inclusione, invece, nell'ambito applicativo della rescissione del giudicato, anch'essa potenzialmente utilizzabile - come vedremo - in adeguamento ai vincoli convenzionali ${ }^{34}$.

Non solo in conseguenza dell'inoppugnabilità "oggettiva" della sentenza di proscioglimento, ma per via dell'intollerabile forzatura sistematica che comporterebbe l'esperibilità in malam partem del rimedio ${ }^{35}$, non c'è alcun margine per la fruibilità della revisione europea su richiesta

32 V. Corte cost., 26 marzo 2015, n. 49, Giurisprudenza costituzionale, 2015, p. 411 , con riguardo ai rapporti fra proscioglimento per prescrizione e confisca.

33 Corte eur., Gr. Camera, 28 giugno 2018, G.I.E.M. s.r.l. e altri c. Italia, §§ 261 ss, in <https://hudoc.echr.coe.int>. Accesso: 13 agosto 2018.

34 Cfr., infra, § 7 .

35 Non è un tabù, naturalmente, porsi sul piano generale il problema dell'introduzione della revisione in peius (sul tema, da ultimo, CORVI, Paola. La revisione in peius. In CORVI, Paola (a cura di). Le impugnazioni straordinarie. Torino: Giappichelli, 2016, p. 110 ss.), ma, invariata la caratura dell'istituto come rimedio straordinario ispirato al favor rei, sarebbe intollerabilmente distonico configurarne un'applicazione in peius nella sola species europea (per ulteriori ragioni ostative v., infra, nel testo). 
della persona offesa ${ }^{36}$, ad esempio in esecuzione di un giudicato convenzionale che - sulla scia di un orientamento affermatosi da tempo a Strasburgo - ravvisi una lesione del "bene vita”, tutelato dall'art. 2 CEDU, nel fatto che l'inerzia degli inquirenti nel perseguire un omicidio abbia determinato un esito proscioglitivo ${ }^{37}$.

La questione è stata espressamente affrontata - e risolta negativamente - nella fase esecutiva della sentenza Alikaj c. Italia, di condanna del nostro Paese per violazione dell'art. 2 CEDU, nei profili sostanziali e processuali, a fronte del proscioglimento per prescrizione, dal reato di omicidio colposo, di un agente di polizia responsabile di avere ucciso un ragazzo durante un inseguimento ${ }^{38}$. Premesso che, nel caso di specie, la sentenza europea non recava alcuna indicazione in ordine alle misure individuali di restitutio in integrum - limitandosi a dichiarare la violazione fra l'altro in quanto «l'applicazione della prescrizione» in siffatte fattispecie «rientra incontestabilmente nella categoria di quelle misure inammissibili, secondo la giurisprudenza della Corte», se abbia «l'effetto di impedire una condanna»-, nell'informare il Comitato dei ministri sulle misure adottate, il Governo italiano escludeva radicalmente la possibilità di riaprire la procedura in questione: ciò in quanto, da un lato, l'ordinamento nazionale non permette l'esperibilità in malam partem della revisione "europea", dall'altro, l'opposta soluzione comporterebbe la lesione della garanzia del ne bis in idem, riconosciuta non solo dalla Costituzione italiana, ma anche dall'art. 4, Prot. 7, $\mathrm{CEDU}^{39}$.

36 In termini dubitativi, invece, con diverse sfumature, GERACI, Rosa Maria. Sentenze, cit., p. 170 ss.; PARLATO, Lucia. Revisione del processo iniquo: la Corte costituzionale "getta il cuore oltre l'ostacolo". Diritto penale e processo, 2011, p. 847; QUATTROCOLO, Serena. Violazioni di convenzioni internazionali e giudicato penale interno. Rivista di diritto processuale, 2012, p. 654 s.

37 Cfr. GERACI, Rosa Maria. L'impugnativa straordinaria per la violazione della Cedu accertata a Strasburgo. Le ipotesi, le procedure, gli effetti. In CORVI, Paola (a cura di). Le impugnazioni straordinarie, cit. p. 77, la quale sottolinea come, proprio in tale caso, l'inesperibilità della revisione europea comporti «un evidente vuoto di tutela».

38 Corte eur., Sez. II, 29 marzo 2011, Alikaj c. Italia, in <https://hudoc.echr.coe. int>. Accesso 13 agosto 2018.

39 Cfr. Il Bilan d'action revisé inviato dal Governo italiano alla segreteria dei Comitato dei ministri il 16 novembre 2015 (DH-DD(2015)1225), in <http:// hudoc.exec.coe.int>. Accesso: 13 agosto 2018. 
Il riferimento all'art. 4, Prot. 7, CEDU richiede qualche precisazione. A norma del § 2 della disposizione, infatti, il ne bis in idem convenzionale non impedisce la riapertura del processo, «conformemente alla legge e alla procedura penale dello Stato interessato», a fronte dell'emersione di fatti nuovi, o di vizi fondamentali della pregressa procedura, che avrebbero potuto condizionarne l'esito, e ciò a prescindere - parrebbe - dal fatto che questo fosse condannatorio o proscioglitivo ${ }^{40}$. Quand'anche, peraltro, possa trarsi da tale previsione l'astratta "compatibilità convenzionale" di ipotesi di revisione sia in melius sia in peius, il testuale richiamo alla legge e alla procedura penale dello Stato interessato va a nostro parere inteso come integralmente "recettivo" delle opzioni di quest'ultimo, e quindi - nel caso dell'Italia - della scelta di circoscrivere il rimedio secondo logiche di favor rei.

Va d'altronde dato atto che, con Risoluzione finale del 24 febbraio 2016, il Comitato dei ministri ha dichiarato positivamente conclusa la procedura esecutiva in re Alikaj, sottolineando come l'Italia abbia adempiuto, per quanto possibile, alle obbligazioni sub art. $46 \mathrm{CEDU}^{41}$.

Né la revisione potrà essere esperita, "agli effetti civili", dalla parte civile che sia riconosciuta vittima, in un processo conclusosi con esito assolutorio, di una violazione del proprio diritto al fair trial: considerato, fra l'altro, che la Corte costituzionale ha dichiarato infondate plurime questioni di legittimità dell'art. 395 c.p.c. in quanto non prevede una "revocazione europea" ${ }^{42}$, sarebbe di assai dubbia razionalità che il danneggiato potesse o meno ottenere la riapertura del processo a seconda della sede in cui agisce, non ravvisandosi alcun fondamento, sotto questo profilo, ad una siffatta disparità di trattamento.

Proprio uno degli argomenti, addotti dal Giudice delle leggi a sostegno delle decisioni da ultimo menzionate, offre del resto ulteriore

40 Cfr. CAVALLARO, Tiziana. La revisione contra reum del giudicato penale tra realtà e prospettive di riforma. Diritto penale e processo, 2010, p. 1112; CORVI, Paola. La revisione in peius, cit., p. 113 s.; MARCHETTI, Maria Riccarda. Commento all'art. 4, legge 9 aprile 1990, n. 88 di ratifica ed esecuzione del Protocollo n. 7 alla Convenzione europea per la salvaguardia dei diritti dell'uomo e delle libertà fondamentali. Legislazione penale, 1991, p. 251 s.; SCALFATI, Adolfo. L'esame sul merito, cit., p. $170 \mathrm{~s}$.

41 Cfr. CM/ResDH(2016)13, in <http://hudoc.exec.coe.int>. Accesso: 13 agosto 2018 .

42 Cfr. Corte cost. 26 maggio 2017, n. 123, cit.; Id., 27 aprile 2018, n. 93, cit. 
conferma alla tesi dell'impossibilità - almeno allo stato - di utilizzare la revisione europea in malam partem, e ciò quand'anche il dato testuale, ricavabile dal dispositivo della sentenza n. 113 del 2011, non sia reputato dirimente, e si ritenga altresì superabile l'ostacolo opposto dal ne bis in idem. Come la Corte costituzionale ha osservato nell'occuparsi della revocazione civile, occorre infatti considerare che il processo "convenzionale" non garantisce il contraddittorio nei confronti di eventuali terzi rispetto al ricorrente e allo Stato convenuto ${ }^{43}$ : ciò posto, si porrebbe certamente in conflitto coi principi di cui agli artt. 24 comma 2 e 111 Cost. la revoca in peius del giudicato nei confronti di un prosciolto - penalmente e/o agli effetti civili - che, non essendo stato parte del giudizio "europeo", non abbia potuto in quella sede impegnarsi per confutare la sussistenza della violazione convenzionale - addotta dalla persona offesa o dal danneggiato - sulla cui base il giudicato dovrebbe essere caducato.

E' vero che l'Explanatory memorandum alla Racc. n. (2000) 2, nel porsi il problema delle third parties che potrebbero essere pregiudicate dalla riapertura o dal riesame della decisione irrevocabile, sottolinea come analogo inconveniente si manifesti nell'ordinario funzionamento "interno" dei rimedi post-iudicatum, e vada quindi gestito nello stesso $\operatorname{modo}^{44}$. Non ci pare, però, che garantire al prosciolto il contraddittorio nel giudizio di revisione promosso dalla persona offesa o dal danneggiato - in applicazione "a parti invertite" dell'art. 636 commi 1 e 2 c.p.p. ${ }^{45}$ - possa bastare a colmare il deficit difensivo in cui questi sia incorso a Strasburgo ${ }^{46}$.

43 A norma dell'art. 44 del Regolamento della Corte, l'intervento di terzi - siano essi Alte parti contraenti o «qualsiasi persona interessata diversa dal ricorrente» - è previsto solo su autorizzazione del Presidente della Camera, il quale «può, nell'interesse di una buona amministrazione della giustizia», invitare tali soggetti «a presentare osservazioni scritte o, in circostanze eccezionali, a prendere parte all'udienza».

44 Cfr. Explanatory Memorandum, cit., § 15.

45 Tali disposizioni, attraverso il rinvio all'art. 601 c.p.p. e alla disciplina del dibattimento in quanto applicabile, prevedono la citazione per il giudizio di revisione di tutte le parti del giudizio a quo.

46 Per un analogo spunto cfr. PADULA, Carlo. La Corte Edu e i giudici comuni nella prospettiva della recente giurisprudenza costituzionale. In <http:// www.giurcost.org/studi/ >, 2016, 11, p. 325. Accesso: 13 agosto 2018. 
A completamento della disamina, in ordine alla legittimazione a proporre la revisione "speciale", occorre ancora chiedersi se l'impugnazione straordinaria sia esperibile da soggetti, diversi dal ricorrente alla Corte europea, che versino in situazione analoga, o finanche identica, a quest'ultimo, ciò che accade quando la violazione, accertata dal giudicato europeo, discenda da un vizio "strutturale" dell'ordinamento interno.

Ad una soluzione positiva del problema osta - sul piano generale - il fatto che la revisione, come attualmente costruita dal Giudice delle leggi, sia strumento di esecuzione del giudicato europeo, come tale strettamente ancorato al dictum della Corte di Strasburgo in ordine alla specifica controversia sottopostale. Ne discende che un obbligo di restitutio in integrum, da eventualmente assolvere tramite un intervento sul giudicato interno, non potrà che riguardare il solo ricorrente alla Corte dei diritti umani, restando fuori dagli effetti "esecutivi" della decisione europea chi versi in posizione analoga, o finanche identica, a costui ${ }^{47}$.

E' pur vero che all'esito di un complesso percorso - che i limiti del presente lavoro non consentono di approfondire - la Corte costituzionale e le Sezioni unite della Cassazione hanno riconosciuto - a certe condizioni - la caducabilità del giudicato anche in favore dei non ricorrenti a Strasburgo, nei soli casi, però, in cui la violazione riscontrata dal giudice europeo, e ascrivibile a un difetto sistemico dell'ordinamento interno, attenga alla materia sostanziale ${ }^{48}$. Nelle medesime decisioni entrambe

47 Cfr., sul piano generale, DE SALVIA, Michele. L'obbligo degli Stati di conformarsi alle decisioni della Corte europea e del Comitato dei ministri del Consiglio d'Europa. In: BALSAMO, Antonio; KOSTORIS, Roberto (a cura di). Giurisprudenza europea e processo penale italiano. Torino: Giappichelli, 2008, p. 72 s. Proprio alla luce dell'argomento evidenziato nel testo, ha espressamente escluso l'ammissibilità della richiesta di revisione proposta da un non ricorrente a Strasburgo, Cass., Sez. II, 20 giugno 2017, n. 40889, Cariolo, in <http://www.italgiure.giustizia.it/sncass/>. Accesso: 13 agosto 2018; v. anche, sempre in termini negativi, GERACI, Rosa Maria. L'impugnativa straordinaria, cit., p. 24. Ritengono invece praticabile un'“estensione soggettiva” della revisione europea FALATO, Fabiana. La relatività del giudicato processuale. Napoli: Editoriale Scientifica, 2016, p. 209 ss.; LORENZETTO, Elisa. Violazioni convenzionali, cit., p. 28 ss.

48 Cfr. Corte cost., 18 luglio 2013, n. 210, Giurisprudenza costituzionale, 2013, p. 2915; Cass., Sez. un., 24 ottobre 2013, Ercolano, Cassazione penale, 2015, p. 28: nella specie, si trattava di rideterminare la pena, calcolata sulla base di una norma applicata in contrasto col criterio della lex mitior ex art. 7 CEDU, 
le Corti hanno invece negato espressamente l'efficacia ultra partes del giudicato europeo accertativo di violazioni processuali, operando un discrimen che - almeno de iure condito - va secondo noi condiviso.

Allorché il problema strutturale ravvisato a Strasburgo verta in materia sostanziale, infatti, l'equivalenza fra le posizioni del ricorrente e dei non ricorrenti al giudice europeo è di accertamento relativamente facile, bastando verificare se la norma sostanziale interna, da cui la violazione convenzionale trae origine, sia stata applicata dal giudice nazionale, ciò che di per sé comporta l'incompatibilità convenzionale dei contenuti decisori del giudicato interno. Al contrario, il fatto che la norma processuale all'origine della violazione sia stata applicata nel processo a carico del non ricorrente, di regola non significa ancora, nella prospettiva convenzionale, che tale processo risulti unfair, e meriti, quindi, la riapertura: nell'accertare l'inosservanza dei canoni del fair trial, invero, la Corte europea opera una valutazione complessiva (as a whole), strettamente ancorata al caso concreto, sì che il medesimo "deficit di garanzia" potrebbe, per soggetti diversi, non sortire effetti analogamente lesivi ${ }^{49}$.

D'altra parte - come abbiamo detto - nel prospettare effetti ultra partes della decisione europea si esorbita dall'ambito strettamente "esecutivo" di quest'ultima, intendendola, piuttosto, alla stregua di un novum ius, che dovrebbe condurre all'interpretazione conforme delle norme processuali interne, o alla deduzione della loro illegittimità costituzionale. Proprio qui, però, si annida un'altra fondamentale differenza fra il giudicato europeo in materia sostanziale e quello che riguardi i canoni processuali: infatti, mentre l'art. 30, comma 4, 1. 11 marzo 1953,

attraverso un incidente di esecuzione. Per maggiori dettagli ci si permette di rinviare a LAVARINI, Barbara. I rimedi post iudicatum alla violazione dei canoni europei. In GAITO, Alfredo (a cura di). I princìpi europei del processo penale. Roma: Dike, 2016, p. 104 ss.

49 Ne prende chiaramente atto Corte cost., 18 luglio 2013, n. 210, cit., nel dire che l'accertamento di "non equità" del processo ex art. 6 CEDU, «vertendo su eventuali errores in procedendo e implicando valutazioni strettamente correlate alla fattispecie specifica, non può che essere compiuto caso per caso, con l'effetto che il giudicato interno può essere posto in discussione soltanto a fronte di un vincolante dictum della Corte di Strasburgo sulla medesima fattispecie». In dottrina sottolinea la tendenziale condivisibilità del discrimen SCIARABBA, Vincenzo. Il giudicato e la Cedu, cit., p. 196 s. 
n. 87 permette di intervenire post iudicatum allorché sia dichiarata incostituzionale una norma penale sostanziale ${ }^{50}$, un analogo intervento non è consentito a fronte dell'annullamento di una norma processuale. Stando così le cose, sarebbe incoerente, oltreché sovversivo del sistema delle fonti, ammettere la revoca del giudicato in caso di "illegittimità convenzionale" di una norma processuale interna, la cui declaratoria di "mera" illegittimità costituzionale non consentirebbe, invece, di superare una decisione irrevocabile ${ }^{51}$.

Un parziale margine di esperibilità della revisione da parte dei non ricorrenti a Strasburgo potrebbe riconoscersi, forse, a fronte della pronuncia, ad opera della Corte europea, di una "sentenza pilota", a norma dell'art. 61, §§ 3 e 4, del Regolamento della stessa Corte ${ }^{52}$. Questa particolare tipologia decisoria, utilizzabile allorché il giudice europeo imputi la violazione convenzionale a un difetto sistemico dell'ordinamento interno, si caratterizza, infatti, per la formulazione nel dispositivo delle prescrizioni necessarie a rimediare e prevenire violazioni potenzialmente seriali, e si rivolge quindi, per definizione, anche a chi, senza avere adito la Corte di Strasburgo, versi nella stessa posizione del ricorrente. In tale situazione, pertanto, la rimozione ultra partes del giudicato tornerebbe a rappresentare un effetto "esecutivo", non meramente riflesso, della decisione di Strasburgo, trovando nuovamente "copertura" nel dispositivo della sentenza costituzionale n. 113 del $2011^{53}$. Al contrario di quanto alcune "avanguardie" giurisprudenziali sostengono ${ }^{54}$, la soluzione non

50 Secondo tale disposizione, «quando in applicazione della norma dichiarata incostituzionale è stata pronunciata sentenza irrevocabile di condanna, ne cessano l'esecuzione e tutti gli effetti penali».

51 Cfr. CAPRIOLI, Francesco. Giudicato e illegalità della pena: riflessioni a margine di una recente sentenza della Corte costituzionale. In: BARGIS, Marta (a cura di). Studi in ricordo di Maria Gabriella Aimonetto. Milano: Giuffré, 2013, p. 285, nota 55; CARNEVALE, Stefania. L'inidoneità dei rimedi individuati dalla giurisprudenza. Diritto penale e processo, 2007, p. 1238.

52 Contra, peraltro, Cass., Sez. II, 20 giugno 2017, n. 40889, Cariolo, cit.

53 Resta tuttavia pressoché proibitivo, a fronte di violazioni convenzionali processuali, accertare l'identità fra le posizioni dei ricorrenti e dei non ricorrenti alla Corte europea.

54 Cfr. Cass., Sez. I, 11 ottobre 2016, n. 44193, D., Cassazione penale, 2017, p. 1374; indirettamente v. anche Cass., Sez. I, 4 luglio 2014, n. 52965, Attanasio, in <http://www.italgiure.giustizia.it/sncass/>. Accesso: 13 agosto 2018. 
sarebbe però estensibile - senza nuovamente esorbitare dall'ambito esecutivo - a decisioni europee che, sebbene evidenzino nella sostanza un problema sistemico, non siano qualificate formalmente, né strutturate nel dispositivo, come "sentenze pilota".

\section{C) I PRESUPPOSTI}

Quanto ai presupposti di esperibilità della revisione europea, una prima questione attiene alla natura delle violazioni convenzionali interessate dal rimedio. Benché, infatti, l'ordinanza di rimessione alla Corte costituzionale sollecitasse una pronuncia additiva con esclusivo riguardo alle violazioni "processuali" ex art. 6 CEDU, nessun richiamo a tale disposizione, e nemmeno al carattere - processuale e/o sostanziale della violazione, compare nel dispositivo della sentenza costituzionale, la cui motivazione si limita d'altronde ad affermare che l'esigenza di riaprire il procedimento - quale presupposto della revisione - andrà apprezzata alla luce vuoi della natura «oggettiva» della violazione, vuoi delle «indicazioni contenute nella sentenza della cui esecuzione si tratta» o nella «sentenza interpretativa» eventualmente sollecitata dal Comitato dei ministri alla Corte di Strasburgo, ex art. 46, § 3, CEDU. Parrebbe quindi che, seppure la revisione europea sia elettivamente deputata al ristoro delle violazioni convenzionali di tipo processuale, non possa escludersene a priori la fruibilità quale rimedio alla violazione di garanzie sostanziali, quando a tal fine occorra intervenire sul contenuto della decisione, ed un diverso e più "snello" rimedio non sia disponibile: del resto, è la stessa Corte costituzionale a qualificare la riapertura del processo un «concetto di genere» idoneo a modellarsi secondo le esigenze del caso concreto, "spaziando" dalla rinnovazione integrale del giudizio alla semplice rivisitazione dell'attività decisoria ${ }^{55}$.

55 Ex plurimis, GERACI, Rosa Maria. L'impugnativa straordinaria, cit., p. 74; GIALUZ, Mitja. Una sentenza "additiva di istituto", cit., p. 3313 s.; LORENZETTO, Elisa. Violazioni convenzionali, cit., p. 14; SCIARABBA, Vincenzo. Il giudicato e la CEDU, cit., p. 253; TROISI, Paolo. Flessibilità del giudicato e tutela dei diritti fondamentali, in <http://www.penalecontemporaneo.it>, 2 aprile 2015, p. 14. Accesso: 13 agosto 2018. In giurisprudenza, Cass., Sez. I, 11 ottobre 2016, n. 44193, D., cit. 
Tralasciando quest'ultimo problema, estraneo all'oggetto del nostro lavoro, deve piuttosto rilevarsi che, da un lato, le garanzie processuali, suscettibili di restitutio in integrum attraverso la revisione europea, non si esauriscono in quelle ex art. $6 \mathrm{CEDU}^{56}$, dall'altro, non tutte le violazioni del fair trial possono trovare rimedio in quest'ultima.

Sotto il primo profilo, la revisione potrebbe ad esempio applicarsi a ristoro della garanzia ex art. 8 CEDU, allorché la Corte europea ne avesse ravvisato la violazione in ragione dell'esperimento, con modalità "convenzionalmente incompatibili", di un mezzo di ricerca probatoria invasivo della sfera privata ${ }^{57}$. A maggior ragione, si potrebbe ricorrere al mezzo straordinario di impugnazione quando la prova fosse stata acquisita violando l'art. 3 CEDU, cioè con sistemi riconducibili alla nozione di tortura o di trattamento inumano o degradante ${ }^{58}$, tantopiù che, in siffatte

56 Per un ampio elenco di precedenti della Corte di Strasburgo ove si è invocata la re-trial clause - nel dispositivo o in motivazione - a fronte della violazione di plurime garanzie ex art 6 CEDU - fra le altre il diritto di accesso alla giustizia, il diritto ad essere giudicati da un giudice costituito per legge, il principio di imparzialità e indipendenza del giudice, il diritto a partecipare al processo, il diritto al confronto coi testimoni, il diritto alla conoscenza dell'accusa e a disporre del tempo e delle condizioni per preparare la difesa, il diritto ad una decisione motivata - cfr. la dissenting opinion del giudice Paulo Pinto de Albuquerque alla sentenza della Grande Camera in re Moreira-Ferreira, cit. (§ 8). Fra le più significative sentenze che hanno riguardato l'Italia, cfr. Corte eur., Sez. II, 18 maggio 2004, Somogyi c. Italia; Id., Gr. Camera, 1 marzo 2006, Sejdovic c. Italia; Id., Sez. III, 8 febbraio 2007, Kollacaku c. Italia; Id., Sez. I, 19 ottobre 2006, Majadallah c. Italia; Id., Sez. II, 12 ottobre 2017, Drassich c. Italia, cit.; Id., Sez. I, 29 giugno 2017, Lorefice c. Italia; Id.; Sez. I, 12 ottobre 2017, Cafagna c. Italia (tutte in <https://hudoc.echr.coe.int>. Accesso: 13 agosto 2018).

QUATTROCOLO, Serena. Violazioni di convenzioni internazionali, cit., p. 654.

Per l'inclusione di siffatta violazione, a titolo esemplificativo, fra quelle suscettibili di riparazione attraverso la riapertura del processo cfr. il § 12 dell'Explanatory memorandum alla Racc. (2000) 2, cit. V., altresì, la Risoluzione del Comitato dei ministri CM/ResDH(2010)53 e relativa Appendice (in <http://hudoc.exec.coe.int/>. Accesso: 13 agosto 2018), concernenti la procedura esecutiva di Corte eur., Gr. Camera, 20 luglio 2006, Jalloh c. Germania (che aveva ravvisato una violazione degli artt. 3 e 6 CEDU nella somministrazione coattiva all'imputato, con modalità violente, di un emetico, al fine di ottenere la prova del reato di detenzione di sostanze stupefacenti): in sede esecutiva lo Stato condannato ha fra l'altro comunicato, quanto alle misure individuali, che, a richiesta del legale del ricorrente vittorioso a Strasburgo, il giudice interno aveva ammesso la riapertura del processo, 
ipotesi, la Corte di Strasburgo riscontra in termini pressoché automatici anche una violazione dell'art. 6 CEDU ${ }^{59}$.

A proposito, invece, delle garanzie del fair trial oggettivamente insuscettibili di restitutio in integrum attraverso la revisione, è la stessa sentenza costituzionale n. 113 del 2011 a ravvisarne un esempio nel diritto dell'accusato a un processo di durata ragionevole, la cui accertata violazione sarebbe addirittura aggravata da un meccanismo di riapertura ${ }^{60}$. Seri dubbi sono stati altresì avanzati sulla necessità della riapertura del procedimento quando sia stata violata la garanzia della pubblicità delle udienze ${ }^{61}$.

Sotto un diverso profilo, occorre considerare che la stessa Corte costituzionale, conscia della natura "emergenziale" del suo intervento additivo, ne segnala la residualità rispetto all'introduzione legislativa di una «diversa disciplina», anche attraverso «un autonomo e distinto istituto ${ }^{62}$. Ciò imporrà di valutare - con un'analisi "dedicata" - se debbano sottrarsi dall'ambito della revisione le violazioni convenzionali relative al processo in absentia, che già all'epoca "coperte" - come riconosciuto dalla stessa Corte - dal rimedio della restituzione in termini, potrebbero ora trovare ristoro nella rescissione del giudicato, introdotta dalla 1.28 aprile 2014 , n. $67^{63}$.

seppure non ne fosse stata successivamente possibile la celebrazione perché l'imputato aveva lasciato la Germania (la cui legge non consente il processo in assenza). A seguito di tale comunicazione, il Comitato dei ministri ha ritenuto positivamente chiusa la procedura esecutiva.

59 Corte eur., Gr. Camera, 20 luglio 2006, Jalloh c. Germania, cit., § 99, nonché, più di recente, Id., sez. I, 30 aprile 2015, Shamardakov c. Russia, in particolare $\S$ 154, e Id., Sez. III, 15 maggio 2018, Sergey Ivanov c. Russia (tutte in <https://hudoc.echr.coe.int>. Accesso: 13 agosto 2018). Per un approfondimento, CASSIBBA, Fabio. Brevi riflessioni sull'inutilizzabilità delle dichiarazioni estorte con tortura ai sensi del nuovo art. 191 comma 2-bis, in <https://penalecontemporaneo.it>, 2018, n. 4, p. 112 s. Accesso: 13 agosto 2018. Corte cost., 7 aprile 2011, n. 113, cit., $§ 8$ del Considerato in diritto. In dottrina, per tutti, GERACI, Rosa Maria. L'impugnativa straordinaria, cit., p. 74.

LOGLI, Andrea. La riapertura del processo a seguito della sentenza Cedu. Questioni interpretative sul nuovo caso di "revisione europea". Cassazione penale, 2012, p. $941 \mathrm{~s}$.

62 Corte cost., 7 aprile 2011, n. 113, cit., $\S 9$ del Considerato in diritto.

63 V. meglio, infra, $\S 7$. 
Fra i criteri, sulla cui base il giudice della revisione dovrà decidere se riaprire il processo, la Corte costituzionale include, ovviamente, le indicazioni del giudicato europeo, delle quali va però chiarita la vincolatività.

Per lo più si esclude che un vincolo, sub specie di radicale impedimento alla riapertura del processo, possa trarsi dall'assenza, nella decisione di Strasburgo, di indicazioni espresse in quest'ultimo senso. Ciò in quanto, da un lato, l'individuazione degli specifici strumenti di restitutio in integrum è comunque rimessa in via principale all'autonomia dello Stato interessato, dall'altro, prescrizioni “esecutive" più dettagliate sono talora impartite non tanto dalla Corte europea - sebbene sempre più attiva in questa direzione -, quanto dal Comitato dei ministri incaricato, ex art. 46, § 2, CEDU, di controllare l'esecuzione delle relative decisioni ${ }^{64}$.

Ci si chiede, però, se il dispositivo della sentenza costituzionale n. 113 del 2011, nel consentire la revisione quando «ciò sia necessario», a norma dell'art. 46 CEDU, per conformarsi al giudicato europeo, richieda un quid pluris, vale a dire la sussistenza, nella decisione di Strasburgo, del "comando" esplicito di riaprire il processo ${ }^{65}$. Ad evitare un'eccessiva discrezionalità del giudice della revisione "europea”, senza però confinare l'istituto entro margini che di fatto ne pregiudichino l'utilità, un equo compromesso può secondo noi raggiungersi limitando l'esperibilità dell'impugnazione alle situazioni in cui la decisione della Corte di Strasburgo, seppure non prescriva la riapertura del processo in termini imperativi indicandola nel dispositivo, o qualificandola in motivazione come il solo rimedio adeguato -, almeno la prospetti in motivazione come rimedio in principio appropriato. La revisione non dovrebbe ammettersi, invece, allorquando né il dispositivo, né la motivazione della decisione europea individuino nel re-trial un idoneo strumento di restitutio in integrum, e ciò anche se un'indicazione in tal senso sia poi formulata dal Comitato dei

64 GERACI, Rosa Maria. Sentenze, cit., p. 160; LOGLI, Andrea. La riapertura del processo, cit., p. 938; LORENZETTO, Elisa. Violazioni convenzionali, cit., p. 12.

65 Per uno spunto in questi termini, con qualche diversa sfumatura, SCIARAB$\mathrm{BA}$, Vincenzo. Il giudicato e la CEDU, cit., p. 252, secondo cui non può «considerarsi sufficiente», per l'ammissibilità dell'istanza di revisione, «la mera circostanza che la Corte di Strasburgo abbia accertato una violazione della CEDU in danno del soggetto che richiede o che comunque beneficerebbe della riapertura». 
ministri nella fase esecutiva ${ }^{66}$. Del resto, come si è giustamente osservato, «un vincolo giuridico a superare» il giudicato «dovrebbe sorgere solo dalla decisione di un organo giurisdizionale», senza alcuna ingerenza - ad evitare una pericolosa "crepa" nel principio di separazione dei poteri - ad opera di un organo di matrice comunque politica ${ }^{67}$.

Va tuttavia fatta salva l'ipotesi in cui l'originario "silenzio" del giudice europeo, in ordine alla necessità od opportunità di caducare il giudicato, sia superato dal medesimo giudice, attraverso la sentenza interpretativa di cui all'art. 46, § 3, CEDU ${ }^{68}$ : è infatti la stessa Corte costituzionale, nel delineare i presupposti di esperibilità della revisione, a valorizzare le indicazioni formulate «nella sentenza interpretativa eventualmente richiesta alla Corte di Strasburgo dal Comitato dei ministri». Col che un chiaro riscontro, fra l'altro, alla tesi - sopra sostenuta - per cui il superamento del giudicato deve fondarsi sulla decisione di un organo giurisdizionale, e non meramente politico.

Laddove il giudicato europeo contenga una re-trial clause, occorrerà peraltro distinguere: se la riapertura del processo sia prescritta nel dispositivo, oppure sollecitata in motivazione in termini tassativi, al giudice delle revisione non potrà riconoscersi alcun margine di scelta in punto ammissibilità del rimedio, ad evitare che il relativo diniego

66 In passato, Cass., Sez. I, 21 febbraio 2008, Rojas, in CED Cass., n. 239987, aveva escluso di poter disporre ex art. 670 c.p.p. la cessazione dell'esecuzione, in adempimento di Corte eur., Sez. II, 16 novembre 2001, Rojas Morales c. Italia, in quanto la decisione europea, pur accertativa della violazione dell'art. 6 CEDU, non aveva espressamente sollecitato la riapertura del processo. In dottrina, sia pure in termini maggiormente dubitativi, GIALUZ, Mitja. Una sentenza "additiva di istituto", cit., p. 3316; LORENZETTO, Elisa. Violazioni convenzionali, cit., p. 12.

67 Così GIALUZ, Mitja. Una sentenza “additiva di istituto”, cit., 3311 s. V. anche, con qualche diverso accento, DE SALVIA, Michele. L'obbligo degli Stati, cit., p. 73; COZZI, Alessia Ottavia. L'impatto delle sentenze della Corte di Strasburgo sulle situazioni giuridiche interne definite da sentenze passate in giudicato: la configurabilità di un obbligo di riapertura o revisione del processo. In: SPITALERI, Fabio (a cura di). L'incidenza del diritto comunitario e della CEDU sugli atti nazionali definitivi. Milano: Giuffré, 2009, p. 175 s.

68 Secondo cui «ove il Comitato dei Ministri ritenga che la sorveglianza di una sentenza definitiva è intralciata dalla difficoltà di interpretare tale sentenza, esso può investire la Corte affinché si pronunci su tale questione d'interpretazione...». 
conduca "in automatico" all'apertura della procedura d'infrazione ex art. 46, §§ 4 e 5, CEDU ${ }^{69}$; qualora, invece, la motivazione della decisione europea qualifichi il re-trial come misura 'in principio' appropriata, o finanche come la misura 'più appropriata', il giudice dell'impugnazione straordinaria godrà di un margine di apprezzamento nel valutarne l'ammissibilità, fermo restando che l'eventuale diniego del rimedio dovrà essere adeguatamente motivato ${ }^{70}$.

A conforto di questa lettura può invocarsi, anzitutto, la prassi del Comitato dei ministri, che ha talora chiuso positivamente la procedura esecutiva, di sentenze recanti una re-opening clause, nonostante le autorità nazionali avessero negato alla vittima della violazione la riapertura del processo, sul presupposto che le ragioni, addotte nel caso concreto a fondamento del diniego, risultassero adeguate ${ }^{71}$.

In termini analoghi si è autorevolmente espressa la Grande Camera in re Moreira-Ferreira. La Corte di Strasburgo era chiamata a verificare la conformità all'art. 6 CEDU del diniego, ad opera del giudice portoghese, della riapertura del processo sollecitata, quale rimedio 'in

69 Secondo cui «ove il Comitato dei Ministri ritenga che un'Alta parte contraente rifiuti di attenersi ad una sentenza definitiva in una controversia di cui è parte, esso può, dopo aver messo in mora questa Parte....investire la Corte dell'osservanza ad opera di questa Parte degli obblighi relativi al paragrafo 1...». In dottrina v. CAIANIELLO, Michele. La riapertura del processo per dare attuazione alle sentenze della Corte europea dei diritti: verso l'affermarsi di un nuovo modello. Quaderni costituzionali, Bologna: Il Mulino, 2011, n. 3 , p. 670, https://doi.org/10.1439/35285, per il quale non vi saranno spazi per rifiutare l'esame dell'istanza di revisione «quando la Corte europea abbia dichiarato iniqua la condanna e abbia chiesto esplicitamente», nonché «in maniera non equivoca», la «riapertura del processo».

70 Su posizioni non dissimili LOGLI, Andrea. La riapertura del processo, cit., p. 939, per il quale devono distinguersi i casi in cui la riapertura «risulti imposta allo Stato», da quelli in cui «questa sia semplicemente "opportuna"».

Cfr. CM/ResDH(2007)1, del 14 febbraio 2007, in <http://hudoc.exec.coe. int/>. Accesso: 13 agosto 2018, con cui il Comitato dei ministri, a chiusura della procedura esecutiva di Corte eur., Gr. Camera, 12 maggio 2005, Ocalan c. Turchia, ha ritenuto che il diniego della riapertura del processo, sollecitata dalla Corte europea a ristoro di plurime violazioni dell'art. 6 CEDU, fosse stato adeguatamente motivato dal giudice nazionale, il quale, considerate le circostanze del caso concreto, aveva escluso che un nuovo giudizio potesse rovesciare gli esiti del primo. In dottrina, CALLARI, Francesco. La revisione, cit., p. 304, nota 119; GIALUZ, Mitja. Una sentenza “additiva di istituto”, cit., p. 3316. 
principio' adeguato, da un precedente giudicato “convenzionale”. La Corte ha condiviso, nel merito, gli argomenti addotti dal giudice nazionale, per il quale la revisione - prevista dall'art. 449 del codice portoghese «when the conviction is irreconciliable with a judgement binding on the Purtuguese State that has been delivered by an international authority or such a judgement casts serious doubt on the validity of the conviction in question» - andava nella specie negata in quanto, «although the procedural irregularity noted by the european Court could have had an impact on the applicant's sentence, it had not been serious enough for the conviction to be considered incompatible with the Court's judgement ${ }^{72}$. Per la Grande Camera, invero, la motivazione fornita dal giudice portoghese - anche alla luce dell'interpretazione, ad opera di costui, del pregresso giudicato europeo - non poteva dirsi arbitraria, ciò bastando, data l'assenza in quel giudicato di un'indicazione vincolante in ordine alla riapertura del processo, ad escludere che il relativo diniego integrasse una nuova violazione del fair trial.

Le considerazioni da ultimo svolte inducono però a riflettere sui parametri che il giudice italiano della revisione, allorché la decisione europea non lo vincoli a riaprire il processo, dovrà applicare nel vaglio preliminare di ammissibilità del rimedio, ex art. 634 c.p.p. ${ }^{73}$

La sentenza costituzionale n. 113 del 2011 afferma espressamente, a segnare il netto distacco della revisione "speciale" da quella tradizionale, che l'istituto di nuovo conio non potrà essere subordinato alla «condizione di ammissibilità, basata sulla prognosi assolutoria, indicata dall'art. 631 c.p.p.» ${ }^{74}$.

Si tratta di una precisazione ineccepibile, dato che - come già rilevato - non necessariamente l'adeguamento al giudicato europeo, in

72 Corte eur., Gr. Camera, 11 luglio 2017, Moreira-Ferreira c. Portogallo, cit., § 88.

73 Nulla quaestio, naturalmente, sul rilievo, agli effetti della valutazione di ammissibilità, dei requisiti formali della richiesta di revisione previsti dall'art. 633 c.p.p., a partire dall'indicazione puntuale e specifica delle ragioni a sostegno dell'impugnazione straordinaria e dall'allegazione, oltreché del giudicato interno di cui si chiede la caducazione, di quello convenzionale su cui la richiesta si fonda.

74 Secondo tale disposizione, «gli elementi in base ai quali si chiede la revisione devono, a pena di inammissibilità della domanda, essere tali da dimostrare, se accertati, che il condannato deve essere prosciolto a norma degli articoli 529,530 о $531 »$. 
particolare se dichiarativo di violazioni convenzionali "processuali", postula una modifica contenutistica di quello interno ${ }^{75}$. Ciò non significa, tuttavia, che la condizione ex art. 631 c.p.p. non possa venire "adattata al ribasso" alle esigenze europee, dando rilievo - nel vaglio di ammissibilità della revisione - quantomeno ad una "aspettativa decisoria” più favorevole, se non ad una vera e propria prognosi assolutoria ${ }^{76}$. E' invero la Racc. n. R (2000) 2 del Comitato dei Ministri - di cui si è già ricordato il ripetuto "avallo" ad opera del giudice di Strasburgo -, ad attribuire peso a siffatta valutazione prognostica, nell'affermare che la riapertura del procedimento andrà circoscritta ai casi, eccezionali, in cui la violazione convenzionale riscontrata sia di tale gravità da indurre seri dubbi sull'esito del processo.

Né potrebbe sostenersi che, poiché l'incidenza causale della violazione sul risultato decisorio è già apprezzata dalla Corte europea, ove quest'ultima, all'esito di siffatto apprezzamento, abbia formulato in motivazione una re-trial clause, il giudice nazionale debba limitarsi a prenderne atto ${ }^{77}$. In particolare nel caso, in cui la violazione convenzionale riscontrata attenga alla prova, deve infatti considerarsi che $i$ parametri, sulla cui base il giudice europeo e quello italiano valutano il "peso" del vizio sulla decisione, sono diversi, giacché il primo decide alla luce della sola sentenza, il secondo anche degli atti processuali: ne discende che una violazione, ritenuta a Strasburgo determinante dell'esito processuale, potrebbe - a ragione - apparire irrilevante al vaglio del giudice interno ${ }^{78}$. Se ne trae conferma dalla già menzionata Risoluzione del Comitato dei ministri a chiusura dell'affaire Ocalan c. Turchia, che ha avallato la decisione del giudice nazionale, di non

75 Cfr., supra, § 2.

76 V. anche, con qualche diversa sfumatura, GIALUZ, Mitja. Una sentenza "additiva di istituto”, cit., p. 3316; LOGLI, Andrea. La riapertura del processo, cit., p. 942; PARLATO, Lucia. Revisione del processo iniquo, cit., p. 846.

77 Lo smentisce apertamente Corte eur., Gr. Camera, 11 luglio 2017, MoreiraFerreira c. Portogallo, cit., § 96, laddove, come appena ricordato nel testo, nemmeno esclude che il giudice nazionale possa autonomamente interpretare - in punto idoneità della violazione ad ingenerare dubbi sulla legittimità della condanna - il decisum di Strasburgo, a condizione che non lo distorca o travisi apertamente.

78 Fra gli altri, GIALUZ, Mitja. Una sentenza “additiva di istituto”, cit., p. 3316; 
dar corso alla riapertura del processo, ritenendone soddisfacente la motivazione, fondata sull'improbabilità di un diverso esito decisorio ${ }^{79}$.

Fra i parametri, che la Racc. n. R (2000) 2 e la giurisprudenza di Strasburgo reputano rilevanti per la decisione sulla riapertura del processo, rientra altresì la serietà delle conseguenze, che la vittima continui a subire per via della violazione convenzionale: nella materia penale si fa l'esempio, in particolare, del perdurante stato detentivo del condannato ${ }^{80}$. Considerato, però, che il dispositivo della sentenza n. 113 del 2011 coinvolge anche il decreto penale di condanna, al quale - per definizione - segue una sanzione esclusivamente pecuniaria (art. 459 comma 1 c.p.p.), è la stessa Corte costituzionale ad escludere che il rimedio resti "confinato" ai casi in cui sia in gioco la libertà personale. Né una condizione, restrittiva dell'accesso alla revisione "speciale", potrebbe ravvisarsi nell'attualità - all'atto della relativa richiesta - degli effetti sanzionatori pregiudizievoli ${ }^{81}$. Poiché, infatti, una simile condizione non è contemplata per la revisione "tradizionale" - che al contrario è garantita «anche se la pena è già stata eseguita o è estinta» (art. 629 c.p.p.) - includerla fra i presupposti del rimedio "europeo" risulterebbe di dubbia compatibilità col principio di legalità processuale.

\section{Il GIUDIZIO DI REVISIONE E I RELATIVI ESITI: LA "TRADUZIONE" DELLE VIOLAZIONI CONVENZIONALI NEI VIZI PROCESSUALI INTERNI}

Posto che obiettivo della revisione "speciale", esperita a ristoro di violazioni convenzionali processuali, è l'integrale o parziale riapertura del processo, non necessariamente la riforma "contenutistica" del giudicato, significativi adattamenti si impongono quanto alle tipologie decisorie conclusive del giudizio sul merito dell'impugnazione straordinaria. E' la stessa Corte costituzionale, del resto, a sancire espressamente l'inapplicabilità alla revisione europea dell'art. 637 comma 2 c.p.p., secondo cui, in caso di accoglimento della richiesta di revisione, la

\footnotetext{
79 Cfr., supra, nota 70.

80 Cfr. l'Explanatory memorandum alla Racc. n. R (2000) 2, cit., § 11.

81 Così, invece, LOGLI, Andrea. La riapertura del processo, cit., p. 640.
} 
corte d'appello, revocato il giudicato di condanna, pronuncia senz'altro sentenza di proscioglimento ${ }^{82}$.

Per individuare le alternative decisorie, a disposizione del giudice della revisione europea, va però risolto il problema della "traduzione" nelle categorie proprie del diritto interno delle violazioni accertate dalla Corte di Strasburgo.

Come il Giudice delle leggi afferma chiaramente, infatti, la più significativa peculiarità della revisione "speciale" è data dal fatto che essa «comporta, nella sostanza, una deroga - imposta dall'esigenza di rispetto degli obblighi internazionali - al principio per cui i vizi processuali restano coperti dal giudicato». Il compito più delicato, che ne deriva al giudice della revisione, consiste allora nel valutare «come le cause della non equità del processo rilevate dalla Corte europea si debbano tradurre... in vizi degli atti processuali alla stregua del diritto interno, adottando nel nuovo giudizio tutti i conseguenti provvedimenti per eliminarli».

La difficoltà di questa operazione nasce dal fatto che, nel sistema italiano, i vizi degli atti processuali sottostanno a un rigido principio di tassatività, sulla cui base, da un lato, un'invalidità va dichiarata allorché prevista dalla legge, a prescindere dall'effettiva lesione degli interessi alla cui tutela è preposta, dall'altro, nessuna invalidità può essere dichiarata nei casi in cui la legge non la preveda, quand'anche una lesione di garanzie difensive si sia, nella sostanza, verificata ${ }^{83}$. Si tratta di una logica completamente antitetica a quella seguita dalla Corte dei diritti umani, per la quale rileva la "sostanza" della violazione di una garanzia convenzionale, a prescindere dal fatto che l'atto processuale, che quella lesione ha determinato, sia stato o no compiuto conformemente alla legge ${ }^{84}$. Se, quindi, la "traduzione" sollecitata dalla Corte costituzionale sarà relativamente semplice quando il dato normativo interno sia capiente rispetto alla riscontrata violazione

82 Corte cost., 7 aprile 2011, n. 113, cit., $\S 8$ del Considerato in diritto.

83 Fra gli altri, CAPRIOLI, Francesco. Abuso del diritto di difesa e nullità inoffensive. Cassazione penale, 2012, p. 2451; VOENA, Giovanni Paolo. Atti. In: CONSO, Giovanni; GREVI, Vittorio; BARGIS, Marta. Compendio di procedura penale. Padova: Cedam, $8^{\text {a }}$ ed., 2016, p. 265.

84 Cfr. KOSTORIS, Roberto. La revisione del giudicato iniquo e i rapporti tra violazioni convenzionali e regole interne. Legislazione penale, 2011, p. 477 ss.; QUATTROCOLO, Serena. Violazioni di convenzioni internazionali, cit., $660 \mathrm{~s}$. 
della CEDU - frutto di mera interpretazione "convenzionalmente difforme" -, rilevanti problemi nasceranno, invece, nell'ipotesi opposta.

Anteriormente all'avvento della revisione europea, una dottrina, quantomeno in relazione alle violazioni dell'art. 6 CEDU, aveva proposto una soluzione radicale al problema che ci occupa. Alla luce dell'art. 111 comma 1 Cost., secondo cui «la giurisdizione si attua mediante il giusto processo...», aveva infatti osservato che la sentenza, frutto di un processo qualificato ingiusto dal giudice convenzionale, potrebbe reputarsi giuridicamente inesistente ${ }^{85}$. Questa opzione, peraltro, appare ormai preclusa proprio dalla scelta del Giudice delle leggi di intervenire in via additiva sulla revisione. L'inesistenza, infatti, è l'unica species di invalidità che, sopravvivendo al giudicato - rectius, impendendone in radice il formarsi ${ }^{86}$ -, non ne postula la revoca, né l'annullamento, ma è di per sé dichiarabile in ogni tempo dal giudice dell'esecuzione, ex art. 670 c.p.p.: se il giudice costituzionale avesse condiviso la menzionata impostazione, sarebbe dunque stata quest'ultima disposizione, non l'art. 630 c.p.p., a doverne raccogliere l'intervento "additivo".

Ciò premesso, si è condivisibilmente suggerito di distinguere, agli effetti della conversione delle categorie convenzionali in quelle interne, le violazioni probatorie - a partire da quelle che traggano origine da una lesione del contraddittorio - dalle violazioni difensive ${ }^{87}$.

a') Quanto alle prime, va considerato che, di regola, la Corte europea non esclude in radice le prove, acquisite in violazione del contraddittorio ex art. 6, § 3, lett. d) CEDU, da quelle fruibili per la decisione, limitandosi per lo più ad impedire che ne rappresentino il fondamento

85 UBERTIS, Giulio. Conformarsi alle condanne europee per violazione dell'equità processuale: doveroso e già possibile. Corriere del merito, 2007, p. 599. V. anche ZACCHÈ, Francesco. Cassazione e iura novit curia, cit., p. 785; MAZZA, Oliviero. L'esecuzione può attendere, cit., p. 2640, nonché, volendo, LAVARINI, Barbara. Giudicato penale, ed esecuzione delle sentenze della Corte europea dei diritti dell'uomo. In: GAMBINI, Rosanna; SALVADORI, Margherita (a cura di). Convenzione europea sui diritti dell'uomo: processo penale e garanzie. Napoli: ESI, 2009, p. 146 s. Su posizioni simili, pur non parlando apertamente di inesistenza, FALATO, Fabiana, La relatività, cit., p. 104 ss.

86 Cfr. VOENA, Giovanni Paolo. Atti, cit., p. 266.

87 Cfr. in particolare KOSTORIS, Roberto. La revisione del giudicato iniquo, cit., p. 477. 
«esclusivo o determinante» ${ }^{88}$. Il giudice, chiamato a riaprire il processo a ristoro di una siffatta violazione, dovrà quindi, se possibile, rinnovare l'assunzione probatoria in termini convenzionalmente compatibili ${ }^{89}$; diversamente - si pensi all'eventualità in cui la violazione consegua all'uso di dichiarazioni oggettivamente irripetibili -, potrà limitarsi a rivalutare il compendio probatorio, comprensivo dell'elemento "contestato" dalla Corte di Strasburgo, nel rispetto della sole or decisive rule ${ }^{90}$. Nessun ostacolo a questa operazione, quali che ne siano gli esiti sulla decisione - che potrebbe essere di proscioglimento o nuovamente di condanna - si trae dall'art. 637 comma 3 c.p.p., secondo cui il giudice non può prosciogliere sulla sola base di una diversa valutazione delle prove pregresse: la stessa sentenza costituzionale n. 113 del 2011, invero, ha escluso espressamente l'applicabilità della disposizione alla revisione europea.

Un'eccezione alla soluzione proposta dovrebbe però ravvisarsi nel caso in cui il diritto interno - "male interpretato" nel giudizio fonte della violazione convenzionale, o medio tempore modificato in termini più garantistici - consenta di ricondurre detta violazione ad una più rigida regola di esclusione probatoria, destinata a prevalere, giusta l'art. 53 CEDU, su quella "valutativa" prescritta dal giudice europeo, e tale da imporre al giudice della revisione una decisione al netto della prova inutilizzabile ${ }^{91}$.

88 Fra le altre, Corte eur., Sez. III, 13 ottobre 2005, Bracci c. Italia, cit.; Id., Sez. I, 19 ottobre 2006, Majadallah c. Italia, cit.; più di recente, per un parziale overruling, v. però Corte eur., Gr. Camera, 15 dicembre 2011, Al Khawaya e Tahery c. Regno unito, in <https://hudoc.echr.coe.int>. Accesso: 14 agosto 2018, secondo cui l'art. 6 CEDU non impedisce radicalmente che la condanna si fondi in modo determinante su una prova acquisita senza contraddittorio, quando siano state riconosciute alla difesa garanzie procedurali "compensative". Sul tema FERRUA, Paolo. La prova nel processo penale, Torino: Giappichelli, 2015, p. 241 ss. Cfr. GIALUZ, Mitja. Una sentenza “additiva di istituto”, cit., p. 3317; KOSTORIS, Roberto. La revisione del giudicato iniquo, cit., p. 477; LORENZETTO, Elisa. Violazioni convenzionali, cit., p. 21. KOSTORIS, Roberto. La revisione del giudicato iniquo, cit., p. 477; v. anche GERACI, Rosa Maria. L'impugnativa straordinaria, cit., p. 85; Sembra invece escludere che l'elemento viziato possa essere rivalutato UBERTIS, Giulio. La revisione successiva a condanne della Corte di Strasburgo. Giurisprudenza costituzionale, 2011, p. 1547.

91 Questa situazione si è registrata nel giudizio di revisione in re Dorigo, nel cui ambito era nato l'incidente di costituzionalità risolto dalla sentenza n. 113 del 2011. C. App. Bologna, 27 gennaio 2014, Dorigo, inedita, ha disposto la 
Qualche precisazione si impone altresì quanto all'ipotesi in cui la violazione accertata a Strasburgo attenga all'art. 3 - oltreché all'art. 6 - CEDU, in ragione di un'acquisizione probatoria in contrasto col divieto di tortura o di trattamenti inumani e degradanti. Delle prove così acquisite, infatti, la giurisprudenza convenzionale preclude l'uso a prescindere da ogni considerazione sul carattere determinante rispetto alla decisione ${ }^{92}$. La via del recepimento interno andrebbe allora individuata nell'art. 191 c.p.p., certamente "capiente" a fronte delle violazioni de quibus, vuoi sulla base del collegamento con la previsione generale ex art. 188 c.p.p., vuoi attraverso il nuovo comma 2-bis, che sancisce la radicale inutilizzabilità delle dichiarazioni acquisite mediante il delitto di tortura ${ }^{93}$. Dichiarata l'inutilizzabilità della prova, il giudice della revisione dovrebbe naturalmente decidere, anche in questo caso, al netto della medesima.

Al novero delle violazioni "probatorie" va ascritta anche l'inosservanza del regime convenzionale dell'overtuning, sulla cui base il prosciolto in primo grado non può - pena la violazione dei canoni del fair trial ex art. 6 CEDU - essere condannato in appello

rinnovazione istruttoria, funzionale all'esame in contraddittorio dei coimputati del Dorigo, illo tempore avvalsisi del diritto al silenzio. Peraltro, in virtù delle modifiche normative sopravvenute al primo "giudicato Dorigo" e alla successiva decisione europea (cfr., supra, nota 15), le dichiarazioni, rese nelle indagini dai coimputati avvalsisi in dibattimento del diritto al silenzio - nei limiti in cui questo possa venire opposto secondo i "novellati" artt. 197 e 197-bis c.p.p. -, sono divenute inutilizzabili verso l'imputato che non abbia potuto esercitare il contraddittorio. In ragione dello ius superveniens, allora, la violazione accertata dalla decisione europea ha trovato specifico riscontro nella categoria interna dell'inutilizzabilità, cioè in una regola di esclusione ben più garantista della sole or decisive rule convenzionale. Nel senso del testo, con qualche diversa sfumatura, KOSTORIS, Roberto. La revisione del giudicato iniquo, cit., p. 477; LORENZETTO, Elisa. Violazioni convenzionali, cit., p. 21; GIALUZ, Mitja. Una sentenza "additiva di istituto", cit., p. 3315.

92 Fra le altre, Corte eur., Gr. Camera, 11 luglio 2006, Jalloh c. Germania, cit., $\S$ 99; Id., Sez. III, 15 maggio 2018, Sergey Ivanov c. Russia, cit.; Id., Sez. I, 30 aprile 2015, Shamardakov c. Russia, cit., § 154. In dottrina CASSIBBA, Fabio. Brevi riflessioni sull'inutilizzabilità, cit., p. 112.

93 Sulla portata, anche nella prospettiva convenzionale, di queste disposizioni, cfr., ancora, CASSIBBA, Fabio. Brevi riflessioni sull'inutilizzabilità, p. $113 \mathrm{~s}$. 
in forza di una mera rivalutazione "cartolare" di prove dichiarative illo tempore assunte ${ }^{94}$.

Come è noto, prima la giurisprudenza di legittimità - attraverso l'interpretazione convenzionalmente conforme dell'art. 603 comma 3 c.p.p. ${ }^{95}$ - poi il legislatore - con l'introduzione dell'art. 603 comma 3-bis ${ }^{96}$ - hanno alfine adeguato la disciplina del giudizio d'appello ai canoni europei. Nelle more di tale percorso, però, l'Italia ha "rimediato" una condanna a Strasburgo, recante in motivazione l'invito a riaprire il procedimento $^{97}$. Nel giudizio di revisione, recentemente promosso sulla scorta del predetto giudicato europeo ${ }^{98}$, la corte d'appello ha correttamente individuato proprio nel novellato art. 603 comma 2-bis c.p.p. lo strumento per "risanare" la violazione convenzionale, disponendo la rinnovazione dell'istruzione dibattimentale ai fini del nuovo esame dei dichiaranti indicati dalla difesa ${ }^{99}$.

In attesa degli esiti dell'impugnazione straordinaria, vale la pena di chiedersi cosa accadrebbe, se la rinnovazione istruttoria risultasse impossibile. Seppure la giurisprudenza convenzionale non sembri

94 Fra le altre, Corte eur., Sez. III, 5 ottobre 2011, Dan c. Moldavia; Id., Sez. III, 4 giugno 2013, Hanu c. Romania, nonché, recentemente, Id., Sez. I, 29 giugno 2017, Lorefice c. Italia (tutte in <https://hudoc.echr.coe.int>. Accesso: 14 agosto 2018).

95 Cass., Sez. un., 28 aprile 2016, Dasgupta, Processo penale e giustizia, 2016, n. 6, p. 46; Id., Sez. un., 28 ottobre 2016, Patalano, Cassazione penale, 2017, p. 2666.

96 Sulla portata della nuova disposizione, introdotta dalla 1. n. 103 del 2017, v., fra gli altri, CAPONE, Arturo. Appello del pubblico ministero e rinnovazione istruttoria. In: BARGIS, Marta; BELLUTA, Hervè (a cura di). La riforma delle impugnazioni tra carenze sistematiche e incertezze applicative, Torino: Giappichelli, 2017, p. 58 ss.

Corte eur., Sez. I, 29 giugno 2017, Lorefice c. Italia, cit., § 52.

Individua nella revisione europea il rimedio ai casi, di violazione del regime convenzionale dell'overtuning, «trattati dalla Corte europea», GALANTINI, Novella. La riassunzione della prova dichiarativa in appello: note a margine di Sezioni unite Troise. In: <http://www.penalecontemporaneo.it>, 17 aprile 2018, p. 8. Accesso: 14 agosto 2018.

Cfr., nell'ambito della procedura esecutiva della sentenza Lorefice c. Italia (DH-DD(2018)36, in <http://hudoc.exec.coe.int/>. Accesso: 14 agosto 2018), la Comunicazione inviata dalle Autorità italiane al Comitato dei Ministri il 20 dicembre 2017, ove si dà conto dell'evolversi del giudizio di revisione. 
imporlo ${ }^{100}$, la conferma del giudicato assolutorio parrebbe inevitabile alla luce della giurisprudenza interna, secondo cui la riassunzione della prova dichiarativa, nel rispetto del principio di oralità/immediatezza, è il solo strumento per superare il "ragionevole dubbio" sulla colpevolezza, ingenerato dal proscioglimento in primo grado: come le Sezioni unite hanno osservato, infatti, tale regola non conosce eccezioni, sì che, quand'anche «la rinnovazione in appello della prova dichiarativa si riveli impossibile, ad esempio per irreperibilità, infermità o decesso del soggetto da esaminare....non vi sono ragioni per ritenere consentito un ribaltamento del giudizio assolutorio ex actis» ${ }^{101}$.

$\mathrm{Al}$ di là della ricordata inapplicabilità dell'art. 637 comma 3 c.p.p., la revisione europea, disposta a ristoro di una violazione convenzionale probatoria, non segna un eccessivo distacco dal modello tradizionale: conformemente a quest'ultimo modello, il giudice dovrà limitarsi a rinnovare i segmenti acquisitivo e/o valutativo del procedimento probatorio - nei termini imposti dall'adeguamento ai canoni europei - senza possibilità di dichiarare d'ufficio l'estinzione del reato ex art. 129 c.p.p., o di rilevare invalidità - estranee alla prova "convenzionalmente viziata" - che non si siano verificate nel medesimo giudizio di revisione ${ }^{102}$. Come nella revisione "ordinaria", ancora, di caducazione del giudicato potrà

${ }^{100}$ La sentenza Lorefice c. Italia ( $\left.\$ 44\right)$, nel dare atto che potrebbero esservi casi - nella specie non dimostrati - in cui «risulta impossibile sentire un testimone di persona nel dibattimento d'appello, ad esempio a causa del suo decesso», richiama, in proposito Corte eur, Gr. Camera, 15 dicembre 2011, Al-Khawaja e Tahery c. Regno unito, cit.: parrebbe allora trarsene che, a fronte dell'impossibilità della riassunzione, il giudice d'appello possa, a norma di Convenzione, condannare l'imputato anche sulla base di una mera rivalutazione cartolare in damnosis della prova dichiarativa, a condizione che questa non sia esclusiva o determinante, o che - secondo appunto la rule Al-Khawaja - possano rinvenirsi garanzie difensive "compensative".

101 Testualmente Cass. Sez. un., 28 aprile 2016, Dasgupta, cit. V. anche Id., Sez. un., 28 ottobre 2016, Patalano, cit.; Id., Sez. Un., 21 dicembre 2017, Troise, in <http:// www.penalecontemporaneo.it>, 17 aprile 2018. Accesso: 14 agosto 2018.

${ }^{102}$ Cfr. GIALUZ, Mitja. Una sentenza “additiva di istituto”, cit., p. 1080, il quale rileva come, in tal caso, la revisione europea si presenti «come impugnazione straordinaria limitatamente devolutiva a struttura rinnovatoria», l'oggetto della quale, «esattamente come [quello del]la revisione ordinaria, è circoscritto entro i limiti segnati dalla richiesta»; v. anche LORENZETTO, Elisa. Violazioni convenzionali, cit., p. 22. 
parlarsi, adattando alle specificità "europee" l'art. 637, comma 2, c.p.p., solo quando la rinnovata assunzione, o valutazione, probatoria, conduca ad esiti decisori diversi e più favorevoli - almeno in parte - rispetto a quelli del processo riaperto. In caso contrario, riacquisirà piena efficacia il "vecchio" giudicato, del quale riprenderà l'esecuzione nel caso in cui il giudice, in applicazione dell'art. 635 c.p.p. ${ }^{103}$ - della cui "compatibilità" con la revisione europea non può dubitarsi ${ }^{104}$ - l'abbia sospesa.

Appaiono di conseguenza adattabili, con qualche forzatura, le categorie decisorie previste per la revisione tradizionale: il giudice accoglierà nel merito l'impugnazione straordinaria ove, alla luce della nuova assunzione o valutazione probatoria, prosciolga l'imputato o lo condanni in termini meno gravosi (art. 637 comma 2 c.p.p.), mentre rigetterà la richiesta di revisione nel caso in cui alla riapertura del processo non segua una modifica in melius del precedente giudicato, bensì la sua "rivitalizzazione" (art. 637 comma 4 c.p.p.). Va da sé che, accedendo a questa ricostruzione, dovrà intendersi fisiologicamente sotteso alla revisione, disposta a ristoro di violazioni probatorie, il divieto di reformatio in peius, peraltro espressamente richiamato, sia pure in un obiter dictum, dalla sentenza n. 113 del $2011^{105}$.

b') E' più complessa la traduzione in vizi processuali interni delle violazioni convenzionali "difensive" (per tali intendendosi, in senso lato, le violazioni dell'art. 6 CEDU che non abbiano direttamente a che fare con la prova ${ }^{106}$ ). Nulla quaestio allorché la violazione trovi riscontro in un'inosservanza di legge interna, e questa sia sanzionata da una nullità speciale - quand'anche non rilevata nel precedente processo -, o possa ricondursi a una nullità generale tramite l'interpretazione convenzionalmente conforme dell'art. 178 c.p.p. ${ }^{107}$

${ }^{103}$ Secondo cui la corte d'appello può sospendere l'esecuzione della pena o della misura di sicurezza, se del caso applicando una fra le misure coercitive ex artt. 281, 282, 283 e 284 c.p.p.

${ }^{104}$ Fra gli altri QUATTROCOLO, Serena. Violazioni di convenzioni internazionali, cit., p. 661.

${ }^{105}$ Corte cost. 7 aprile 2011, n. 113, cit., $§ 5$ del Considerato in diritto.

${ }^{106} \mathrm{Si}$ pensi ad un processo celebrato davanti a un giudice non indipendente o imparziale, oppure alla violazione dei diritti di informativa o di altre garanzie partecipative dell'imputato.

${ }^{107}$ Cfr. GERACI, Rosa Maria. L'impugnativa straordinaria, cit., p. 85. 
Quid iuris, invece, se l'atto processuale interno, in cui la Corte europea abbia ravvisato una violazione convenzionale, sia stato inequivocabilmente compiuto conformemente alla legge ${ }^{108}$ ? In tale caso il principio di tassatività delle invalidità non lascia alcuno spazio d'interpretazione conforme, e non sembra quindi residuare altra via che un incidente di costituzionalità, fondato sul contrasto fra la norma nazionale e gli artt. 6 CEDU e 117 Cost. ${ }^{109}$

Non convince la diversa impostazione per cui sarebbero comunque applicabili le fattispecie di nullità generale, sul presupposto che la violazione di legge, richiesta dagli artt. 177 e 178 c.p.p. per il concretarsi della nullità, sia riferibile all'art. 6 CEDU nell'interpretazione offertane dalla giurisprudenza europea ${ }^{110}$ : siffatta lettura si tradurrebbe infatti in un'intollerabile disapplicazione della legge nazionale, laddove esclude la nullità di un atto conforme al suo modello legale, in favore dell'applicazione diretta di una regola europea di matrice giurisprudenziale, operazione radicalmente esclusa - questa - dalla stessa giurisprudenza ordinaria e costituzionale $^{111}$, e comunque in contrasto con l'art. 101 comma 2 Cost. ${ }^{112}$

Nei limiti in cui la violazione convenzionale trovi riscontro in una nullità interna, il giudice della revisione dovrà dichiararla, traendone tutte

108 Per un calzante esempio v. KOSTORIS, Roberto. La revisione del giudicato iniquo, cit., p. 479, il quale ipotizza che «venga concesso il termine prescritto dalla legge, ma questo si riveli, nel caso concreto, del tutto inadeguato a una difesa effettiva», conducendo la Corte europea a ravvisare la violazione dell'art. $6, \S 1$, lett. b) CEDU: in tale eventualità, la pur ampia fattispecie di nullità generale ex art. 178 lett. c) c.p.p. non potrebbe certo invocarsi, non essendo ravvisabile alcuna «inosservanza delle disposizioni stabilite dalla legge per gli atti del procedimento».

${ }^{109}$ KOSTORIS, Roberto. La revisione del giudicato iniquo, cit., p. 479; UBERTIS, Giulio. La revisione, cit., p. 1547.

${ }^{110}$ Cfr. IACOVIELLO, Francesco Maria. Il quarto grado, cit., p. 815.

${ }^{111}$ Cfr., in termini particolarmente chiari, Cass., Sez. Un., 28 aprile 2016, Dasgupta, cit.: «i principi contenuti nella Convenzione europea per la salvaguardia dei diritti dell'uomo e delle libertà fondamentali, come viventi nella giurisprudenza consolidata della Corte EDU, pur non traducendosi in norme di diretta applicabilità nell'ordinamento nazionale, costituiscono criteri di interpretazione ('convenzionalmente orientata') ai quali il giudice nazionale è tenuto a ispirarsi nell'applicazione delle norme interne».

${ }^{112}$ In termini critici verso questa soluzione interpretativa KOSTORIS, Roberto. La revisione del giudicato iniquo, cit., p. 479 s. 
le conseguenze che il regime di tale invalidità impone, giusta gli artt. $185 \mathrm{e}$ 604 c.p.p. ${ }^{113} \mathrm{~A}$ fronte della nullità di un atto propulsivo, quindi, la revisione europea finirà per avere natura pienamente rescindente, con quanto ne deriva in termini di immediata caducazione del giudicato - quale effetto automatico del vaglio positivo sull'ammissibilità dell'impugnazione -, di eventuale regressione del procedimento ${ }^{114}$, e di reviviscenza, in capo al giudice procedente, di pieni poteri cognitivi e decisori, anche agli effetti del proscioglimento immediato ex art. 129 c.p.p. ${ }^{115}$

Taluno contesta questa soluzione, rilevando che, proprio in ragione dell'innesto del nuovo rimedio sugli artt. 629 ss. c.p.p., il giudice della revisione europea non potrà esercitare poteri diversi da quelli strettamente necessari a rimediare alla violazione convenzionale, con esclusione, in particolare, del potere di pronunciare d'ufficio il proscioglimento ex art. 129 c.p.p. o di rilevare altre invalidità incorse - e non dichiarate - nel giudizio di cognizione, ove la Corte di Strasburgo non le abbia ritenute "convenzionalmente rilevanti"116.

A noi sembra però che, in difetto di diversa e apposita disciplina, alla declaratoria di una nullità - quand'anche effettuata dal giudice della revisione per "tradurre" nelle categorie interne una violazione convenzionale - non possano che seguire gli effetti che la stessa produrrebbe nell'ordinario giudizio di cognizione. Riconoscendo alla revisione europea,

113 Cfr. UBERTIS, Giulio. La revisione, cit., p. 1547.

${ }^{114}$ Contra, limitatamente a questo profilo, LORENZETTO, Elisa. Violazioni convenzionali, cit., p. 23.

115 V. GIALUZ, Mitja. Una sentenza “additiva di istituto", cit., p. 3317; LORENZETTO, Elisa. Violazioni convenzionali, cit., p. 23; MAZZA, Oliviero. La procedura penale, cit., p. 50. Resta da chiedersi entro che limiti il giudice procedente, a norma appunto dell'art. 129 c.p.p., possa dichiarare il reato estinto per prescrizione: secondo GIALUZ, Mitja. Una sentenza "additiva di istituto", cit., p. 3317, nota 27 , «il tempo compreso tra la pronuncia della sentenza di condanna irrevocabile e la sua revoca, ossia lo spatium temporis rappresentato dal giudizio davanti alla Corte di Strasburgo sino all'ammissione della revisione europea» non va computato nel termine di prescrizione. Auspica un intervento legislativo chiarificatore QUATTROCOLO, Serena. Violazioni di convenzioni internazionali, cit., p. $665 \mathrm{~s}$.

${ }^{116}$ Cfr., con varie sfumature, KOSTORIS, Roberto. La revisione del giudicato iniquo, cit., p. 478; QUATTROCOLO, Serena. Violazioni di convenzioni internazionali, cit., p. 662 ss. 
deputata a riaprire il processo a ristoro di una violazione difensiva inficiante un atto propulsivo, effetto pienamente rescindente, si evita inoltre un irragionevole squilibrio fra tale rimedio e la rescissione del giudicato, che anch'essa fruibile - come vedremo - per il ripristino di una garanzia convenzionale di siffatta natura, ha effetti ampiamente restitutori ${ }^{117}$.

Va da sé che, nell'eventualità che ci occupa, le tipologie decisorie conclusive del giudizio di revisione saranno decisamente eccentriche rispetto ai modelli ex art. 637 c.p.p., registrandosi la difficoltà, in particolare, di distinguere nettamente il piano dell'ammissibilità da quello del merito ${ }^{118}$ : al giudice della revisione sembra porsi, invero, la sola alternativa fra la declaratoria di inammissibilità dell'impugnazione straordinaria, e il relativo accoglimento per il tramite di una sentenza, meramente rescindente, assimilabile a quella di annullamento, a cui segua la trasmissione degli atti al giudice di primo o secondo grado, secondo i criteri ex art. 604 c.p.p. L'“aggancio" testuale per questa soluzione è secondo noi rinvenibile nel già ricordato art. 185 c.p.p., quale norma di portata generale applicabile, per effetto della caducazione del giudicato, anche nel giudizio di revisione. La clausola di salvaguardia ex art. 185 comma 3 c.p.p., in una con il fatto che giudice della revisione è la corte d'appello, può poi giustificare - sia pure con qualche forzatura - il richiamo all'art. 604 c.p.p. ${ }^{119}$

Resta da chiedersi se nel nuovo giudizio, seguente all'annullamento del giudicato affetto da violazioni convenzionali "difensive", viga il divieto di reformatio in peius. In senso almeno in parte contrario depone un noto precedente delle Sezioni Unite, che, seppure riconosca nel divieto un «principio di carattere generale in materia di impugnazioni», ne esclude l'operatività a fronte di una sentenza di annullamento ex art. 604 comma 4 c.p.p., cioè di una sentenza che dichiari una nullità assoluta, $\mathrm{o}$ intermedia non sanata, da cui sia derivata la nullità del decreto che dispone il giudizio o della sentenza di primo grado ${ }^{120}$. A fondare la

${ }^{117}$ Cfr., infra, $\S 7$.

118 V. anche QUATTROCOLO, Serena. Violazioni di convenzioni internazionali, cit., p. 660 e 662.

${ }^{119}$ Criticamente, invece, QUATTROCOLO, Serena. Violazioni di convenzioni internazionali, cit., p. $662 \mathrm{~s}$.

${ }^{120}$ Cass., Sez. un., 11 aprile 2006, Maddaloni, CED Cass., n. 233729, secondo cui «il concetto di reformatio in peius implica necessariamente l'esistenza di un 
soluzione positiva può però invocarsi il già ricordato obiter dictum della sentenza costituzionale n. 113 del $2011^{121}$, oltre alla dubbia conciliabilità di una revisione in damnosis, da un lato, col requisito dell'interesse a impugnare che sorregge anche le impugnazioni straordinarie, dall'altro, con quella prognosi di "decisione più favorevole" che, anche sulla scorta dei criteri ricavabili dalla giurisprudenza convenzionale e dalla prassi del Comitato dei Ministri, abbiamo detto condizionare l'accesso all'istituto.

\section{I CONFINI TRA LA REVISIONE "EUROPEA" E LA RESCISSIONE DEL GIUDICATO}

Come abbiamo accennato, 1'“addizione" al sistema della revisione europea è stata giustificata dalla Corte costituzionale a titolo di mera supplenza di un auspicabile intervento del legislatore, «ovviamente libero di regolare con una diversa disciplina - recata anche dall'introduzione di un autonomo e distinto istituto - il meccanismo di adeguamento alle pronunce definitive della Corte di Strasburgo ${ }^{122}$.

Lo strumento apprestato dalla sentenza n. 113 del 2011 non potrà quindi applicarsi a ristoro delle violazioni del fair trial che possano contare su un diverso rimedio, predisposto, appunto, per via legislativa.

Questo sembra il caso delle violazioni connesse al processo in absentia, per fare fronte alle quali il legislatore, proprio sulla scia delle plurime condanne in cui l'Italia era incorsa a Strasburgo - e dunque in adeguamento "strutturale" ai canoni convenzionali - aveva prima introdotto una fattispecie speciale di restituzione in termini ${ }^{123}$, poi sostituita - nel contesto della radicale riforma del giudizio in assenza ad opera della 1. 28

termine di paragone rappresentato da una precedente sentenza, presupposto che viene a mancare quando questa sia stata cancellata, in quanto atto finale di un giudizio nullo e perciò privo di effetti». Proprio su tali basi, adombra l'inoperatività del divieto QUATTROCOLO, Serena. Violazioni di convenzioni internazionali, cit., p. 662 e nota 36.

121 Cfr., supra, nota 100.

${ }^{122}$ Corte cost., 7 aprile 2011, n. $113 \S 9$ del Considerato in diritto.

${ }^{123}$ Ci riferiamo al d.1. 21 febbraio 2005, n. 17, conv. in 1. 22 aprile 2005, n. 60, che, modificando l'art. 175 comma 2 c.p.p., aveva ampliato i margini della restituzione nei termini per impugnare la sentenza contumaciale. 
aprile 2014, n. 67 - dalla rescissione del giudicato. Quest'ultima, originariamente attribuita alla competenza funzionale della Corte di cassazione e disciplinata dall'art. 625-ter c.p.p., è stata "trasferita" dalla l. n. 103 del 2017 alla competenza della corte d'appello ${ }^{124}$, e ricollocata sistematicamente nell'art. 629-bis c.p.p., vale a dire nell'alveo della disciplina della revisione, in cui trova "albergo" anche la variante europea dell'istituto ${ }^{125}$.

Se quindi la Corte dei diritti umani ravvisasse, oggi, una violazione del regime convenzionale del processo in assenza - magari per i rigidi oneri probatori a cui i rimedi restitutori ante-iudicatum, e la stessa rescissione quale ultima via di ricorso interno ex art. $35 \mathrm{CEDU}^{126}$, sono condizionati $^{127}$-, l'esecuzione dei relativi dicta dovrebbe passare proprio

${ }^{124}$ Da individuarsi, sotto il profilo territoriale, in quella «nel cui distretto ha sede il giudice che ha emesso il provvedimento» (art. 629-bis comma 2 c.p.p.) e non, come nel caso della revisione, in quella identificata «secondo i criteri dell'articolo 11» (art. 633 comma 1 c.p.p.).

125 Per un completo quadro della rescissione del giudicato, a partire dall'introduzione sino alle più recenti modifiche, v. SPAGNOLO, Paola. Un istituto nuovo e uno vecchio: la rescissione del giudicato e la restituzione nel termine. Legislazione penale, 2014, n. 4, p. 636 ss.; SPAGNOLO, Paola. La rinnovata fisionomia della rescissione del giudicato. In: BARGIS, Marta; BELLUTA, Hervè (a cura di). La riforma delle impugnazioni, cit., p. 141 ss.

${ }^{126}$ Cfr. DIDDI, Alessandro. Novità in materia di impugnazioni e di restitutio in integrum. In: VIGONI, Daniela (a cura di), Il giudizio in assenza dell'imputato. Torino: Giappichelli, 2014, p. 224, secondo cui la richiesta di rescissione rappresenta «il nuovo rimedio interno di cui il condannato che ritenga di aver subito un processo in absentia in violazione dell'art. 6 della CEDU dovrà servirsi prima di rivolgersi alla Corte di Strasburgo» (non risultando altrimenti esaurite le vie di ricorso interne, ex art. 35 CEDU). V. anche LORENZETTO, Elisa. Violazioni convenzionali, cit., p. 24; SPAGNOLO, Paola. Un istituto nuovo e uno vecchio, cit., p. 638.

127 Spetta infatti all'imputato o al condannato "in assenza" provare che questa è stata dovuta «ad una incolpevole mancata conoscenza della celebrazione del processo» (art. 629-bis comma 1 c.p.p.) in ciò ravvisandosi un nuovo «spazio di possibile "sofferenza convenzionale"»: testualmente, QUATTROCOLO, Serena. Il contumace cede la scena processuale all'assente, mentre l'irreperibile l'abbandona. Diritto penale contemporaneo, Riv. trim, 2014, n. 2, p. 105 s. (disponibile in <http://www.penalecontemporaneo.it>. Accesso: 14 agosto 2018); v. altresì, anche per l'individuazione di ulteriori profili di attrito col regime convenzionale dell'assenza, CASIRAGHI, Roberta. La rescissione del giudicato: molte questioni interpretative sul tappeto. Rivista italiana di diritto e procedura penale, 2018, p. 207 ss. Per un completo quadro delle coordinate convenzionali del processo in assenza QUATTROCOLO, Serena. Assenza e 
dall'istituto "speciale" ex art. 629-bis c.p.p., non dalla revisione europea, quale rimedio di portata generale e suppletiva ${ }^{128}$.

L'assunto non è condiviso da una parte della dottrina, secondo cui la rescissione «ad ora...nulla [ha] a che fare con l'esecuzione delle sentenze della Corte di Strasburgo, nel senso che non ne rappresenta uno strumento di attuazione» ${ }^{129}$; anche a ristoro di violazioni convenzionali in tema di processo in absentia, quindi, «non resterebbe che affidarsi alla revisione europea..... ${ }^{130}$.

Non condividiamo questa tesi. Va rimarcato, anzitutto, come la rescissione prescinda dall'invalidità della decisione in assenza, dato che viene garantita al condannato (o al prosciolto sottoposto a misura di sicurezza), che dimostri l'incolpevole ignoranza del processo, non solo quando questo sia stato illegittimamente celebrato fuori dei casi ex art. 420-bis c.p.p., ma anche quando, ricorrendo gli indici di conoscenza del procedimento ivi previsti, il giudizio in absentia fosse legittimo ${ }^{131}$. Si tratta di un'impostazione pienamente conforme - almeno sotto questo profilo - alla logica convenzionale, che, come ribadito più volte, svincola

irreperibilità dell'imputato. Enciclopedia del diritto, Annali, IX. Milano: Giuffrè, 2016, p. 31 ss..

${ }^{128}$ Così anche DIDDI, Alessandro. Novità in materia di impugnazioni, cit., p. 224; MAZZA, Oliviero. Cedu e diritto interno. In: GAITO, Alfredo. I princìpi europei del processo penale, cit., p. 17 s.; TROISI, Paolo. La flessibilità, cit., p. 15, nota 56. Con maggiori riserve LORENZETTO, Elisa. Violazioni convenzionali, cit., p. 24.

129 Testualmente BELLUTA, Hervè. Le impugnazioni come rimedi ripristinatori: verso il giusto processo in assenza dell'imputato. In: DANIELE, Marcello; PAULESU, Pier Paolo (a cura di). Strategie di deflazione penale e rimodulazioni in absentia. Torino: Giappichelli, 2015, p. 267; v. anche SPAGNOLO, Paola. Un istituto vecchio e uno nuovo, cit., p. 638, nota 10.

${ }^{130}$ CASIRAGHI, Roberta. La rescissione del giudicato, cit., p. 210.

${ }^{131} \mathrm{Cfr}$., con qualche diversa sfumatura, BARGIS, Marta. La rescissione del giudicato ex art. 625 ter c.p.p.: un istituto da rimeditare, Diritto penale contemporaneo, Riv. trim., 2015, n. 1, p. 165 (disponibile in <http://www.penalecontemporaneo.it>. Accesso: 14 agosto 2018); BELLUTA, Hervè. Le impugnazioni come rimedi ripristinatori, cit., p. 268; DI PAOLO, Gabriella. I presupposti della rescissione del giudicato. In: CORVI, Paola (a cura di). Le impugnazioni straordinarie, cit., p. 216; LORENZETTO, Elisa. Soltanto in Cassazione o anche in appello, questo è il dilemma, in <http://www.lalegislazionepenale. eu/>, 7 dicembre 2017, p. 10 e nota 29. Accesso: 14 agosto 2018; QUATTROCOLO, Serena. Assenza e irreperibilità, cit., p. 54 . 
l'ingiustizia dall'invalidità del processo. Sarebbe allora paradossale negare la fruibilità, in adeguamento al giudicato europeo, proprio dell'unico rimedio che, diversamente dalla revisione, non chiede di mettere in discussione il principio di tassatività delle invalidità, per estendere queste ultime ad atti conformi alla legge, ma convenzionalmente viziati.

A ciò si aggiunga che la rescissione offre un modulo procedimentale - incentrato su una prima fase rescindente, cui segue la trasmissione degli atti al giudice di primo grado per il giudizio di merito - di per sé idoneo a garantire una pressoché integrale rinnovazione processuale, laddove - per raggiungere analogo obiettivo - la revisione europea, come si è detto, postula una forzatura del modello ex artt. 634-636 c.p.p.

Va da sé che l'esperibilità del rimedio in chiave riparatoria della violazione convenzionale rischia di trovare ostacolo in quella stessa rigidità dei relativi presupposti - a partire dall'onere di provare «che l'assenza è stata dovuta a un'incolpevole mancata conoscenza del processo» - che, come accennato, potrebbe impedire alla rescissione di prevenire quella violazione. In tale eventualità, peraltro, ammesso che fallisca ogni tentativo d'interpretazione conforme ${ }^{132}$, o che ad assolvere l'onus probandi non possa bastare l'allegazione del giudicato europeo, la soluzione corretta sarebbe un incidente di costituzionalità dell'art. 629-bis c.p.p. ${ }^{133}$.

Ciò premesso, qualche riflessione è ancora opportuna sull'ambito applicativo e sui profili procedimentali della rescissione declinata in chiave europea, onde metterne meglio in luce analogie, differenze e interazioni con la revisione "speciale". Sotto questo profilo, va innanzitutto sottolineato che la rescissione, anche se "spesa" in esecuzione di un giudicato europeo, non può essere condizionata - diversamente dalla revisione "speciale" - ad alcuna prognosi di miglior esito decisorio del nuovo processo, non essendovi traccia di tale condizione nell'art. 629-bis c.p.p. ${ }^{134}$ Data l'assoluta preminenza del diritto di partecipazione

${ }^{132}$ Per un siffatto tentativo cfr. CASIRAGHI, Roberta. La rescissione del giudicato, cit., p. 210 ss.; SPAGNOLO, Paola. La rinnovata fisionomia della rescissione, cit., p. 159 s.

${ }^{133}$ Cfr. LORENZETTO, Elisa. Violazioni convenzionali, cit., p. 24.

${ }^{134}$ In ordine all'assenza, fra le condizioni di ammissibilità della rescissione, di una prognosi sull'esito del processo, v. SPAGNOLO, Paola. Un istituto nuovo e uno vecchio, cit., p. 647. 
personale al processo nell'ambito delle garanzie del fair trial, richiedere in concreto una siffatta valutazione potrebbe del resto apparire eccessivo, ben potendosi presumere - sino a successiva smentita - che proprio la compressione delle garanzie partecipative dell'imputato abbia influenzato negativamente gli esiti del primo giudizio.

Non è chiaro, piuttosto, se il giudice della fase rescissoria sia o no vincolato al divieto di reformatio in peius. La dottrina occupatasi dell'ambito "fisiologico" del rimedio ex art. 629-bis c.p.p. ha opinioni discordanti ${ }^{135}$. A nostro parere, in favore dell'operatività del divieto potrebbe pesare, in una con la sua natura di principio generale delle impugnazioni, il fatto che - coerentemente all'applicabilità della rescissione anche fuori dei casi di invalidità del processo in assenza - il provvedimento rescindente si traduca nella revoca, non nell'annullamento, del pregresso giudicato: ciò dovrebbe escludere l'estensibilità all'istituto di quell'orientamento giurisprudenziale - già ricordato in materia di revisione "speciale" 136 - per cui l'annullamento della decisione impugnata, a fronte della nullità non sanata di un atto propulsivo, elide il divieto di reformatio in peius ${ }^{137}$. Con specifico riguardo alla fruibilità della rescissione in chiave esecutiva di un giudicato europeo, pare altresì richiamabile - per coerenza sistematica la presa di posizione della Corte costituzionale in ordine all'applicabilità del divieto alla revisione "speciale" 138 .

135 In senso contrario all'operatività del divieto v. BARGIS, Marta. La rescissione del giudicato, cit., p. 168; BELLUTA, Hervè. Le impugnazioni come rimedi ripristinatori, cit., p. 273; CARVELLI, Chiara. Rescissione del giudicato e reformatio in peius. Diritto penale e processo, 2014, p. 1045 s.; CASIRAGHI, Roberta. La rescissione del giudicato, cit., p. 237; FALATO, Fabiana. La relatività del giudicato, cit., p. 357; SPAGNOLO, Paola. La rinnovata fisionomia della rescissione, cit., p. 163. Favorevolmente, invece, BISCARDI, Giuseppe. Eclissi della contumacia e sospensione per irreperibilità, tra conoscenza legale e conoscenza reale del processo. Processo penale e giustizia, 2014, n. 6, p. 119 s. (disponibile in <http://www.processopenaleegiustizia.it/>. Accesso: 14 agosto 2018); CONTI, Giovanni. Il processo in absentia: le ricadute sul giudicato, in <http://www.penalecontemporaneo.it>, 2 marzo 2015, p. 5. Accesso: 14 agosto 2018.

${ }^{136}$ Cfr., supra, § 6 e nota 119.

137 V. anche CONTI, Giovanni. Il processo in absentia, cit., p. 5.

${ }^{138}$ Cfr. BISCARDI, Giuseppe. Eclissi della contumacia, cit., p. 120; CONTI, Giovanni. Il processo in absentia, cit., p. 5. 


\section{Spunti de iURE CONDENDO}

Una riflessione si impone, da ultimo, sulle potenzialità espansive della rescissione del giudicato, che quale strumento, appunto, meramente rescindente, cui segue la trasmissione degli atti al giudice di primo grado, potrebbe offrire congruo rimedio - senza forzare la disciplina della revisione - alle violazioni convenzionali che, come quella del diritto dell'imputato a partecipare al processo, impongano la rinnovazione in toto di quest'ultimo (si pensi, in particolare, ai difetti di imparzialità e indipendenza del giudice).

La Relazione alla p.d.l. poi sfociata nella 1. n. 67 del 2014 includeva, invero, un cenno all'estensione dell'ambito del rimedio, nel dire che sulla rescissione «si potranno poi innestare...i casi di processo dichiarato ingiusto dalla Corte europea dei diritti umani» ${ }^{139}$. Un tale ampliamento appare però prospettabile solo de iure condendo, data l'inequivoca limitazione della rescissione alle sole violazioni difensive collegate al rito in absentia.

In definitiva, si dovrebbe ragionare sulla possibilità di "fondere" in un solo rimedio la revisione europea e la rescissione del giudicato ${ }^{140}$, demandando alla corte d'appello di decidere, alla luce della violazione convenzionale riscontrata dalla Corte di Strasburgo: a) se il giudicato interno vada revocato; $b$ ) se al provvedimento revocatorio debba seguire la trasmissione degli atti al giudice di primo grado, per una completa rinnovazione del giudizio "convenzionalmente viziato", o la "ritenzione" del giudizio di merito davanti alla stessa corte d'appello, onde parzialmente ripetere le attività istruttorie o decisorie. In alternativa, la fase rescindente potrebbe essere affidata alla Cassazione, ciò che avrebbe il vantaggio di accentrare, in funzione nomofilattica, l'elaborazione

${ }^{139}$ Cfr. la Relazione alla p.d.l. n. 331/C, presentata alla Camera dei Deputati il 18 marzo 2013, p. 8, reperibile in <http://documenti.camera.it/_dati/leg17/lavori/stampati/pdf/17PDL0001000.pdf>. Accesso: 14 agosto 2018. A favore dell'estensione TONINI, Paolo; CONTI, Carlotta. Il tramonto della contumacia, l'alba radiosa della sospensione e le nubi dell'assenza "consapevole". Diritto penale e processo, 2014, p. 509; esprimono invece riserve BARGIS, Marta. La rescissione del giudicato, cit., p. 162; BELLUTA, Hervè. Le impugnazioni come rimedi ripristinatori, cit., p. 267.

${ }^{140}$ Suggerisce di riflettere «circa le interazioni» tra i due rimedi LORENZETTO, Elisa. Violazioni convenzionali, cit., p. 23. 
dei presupposti per la caducazione del giudicato ${ }^{141}$, ma rischierebbe sul fronte opposto - di accrescere nuovamente i carichi del giudice di legittimità ${ }^{142}$, compromettendo, paradossalmente, proprio l'adeguato esercizio di tale funzione.

\section{Bibliografia}

AIMONETTO, Maria Gabriella. Condanna "europea" e soluzioni interne al sistema processuale penale: alcune riflessioni e spunti de iure condendo. Rivista italiana di diritto e procedura penale, 2009, p. 1510.

BARGIS, Marta. La rescissione del giudicato ex art. 625 ter c.p.p.: un istituto da rimeditare, Diritto penale contemporaneo, Riv. trim., 2015, n. 1, p. 160 (disponibile in <http://www.penalecontemporaneo.it>. Accesso: 14 agosto 2018)

BELLUTA, Hervè. Le impugnazioni come rimedi ripristinatori: verso il giusto processo in assenza dell'imputato. In: DANIELE, Marcello; PAULESU, Pier Paolo (a cura di). Strategie di deflazione penale e rimodulazioni in absentia. Torino: Giappichelli, 2015, p. 249.

BISCARDI, Giuseppe. Eclissi della contumacia e sospensione per irreperibilità, tra conoscenza legale e conoscenza reale del processo. Processo penale e giustizia, 2014, n. 6, p. 103 (disponibile in <http://www.processopenaleegiustizia.it/>. Accesso: 14 agosto 2018)

CAIANIELLO, Michele. La riapertura del processo per dare attuazione alle sentenze della Corte europea dei diritti: verso l'affermarsi di un nuovo modello. Quaderni costituzionali. Bologna: Il Mulino, 2011, n. 3, p. 670, https://doi.org/10.1439/35285

CALLARI, Francesco. La revisione. La giustizia penale tra forma e sostanza. Torino: Giappichelli, 2012.

CAPONE, Arturo. Appello del pubblico ministero e rinnovazione istruttoria. In: BARGIS, Marta; BELLUTA, Hervè (a cura di). La riforma delle impugnazioni tra carenze sistematiche e incertezze applicative, Torino: Giappichelli, 2017, p. 53.

${ }^{141}$ Un simile auspicio, in prospettiva di riforma della revisione "europea", è stato espresso da GIALUZ, Mitja. Una sentenza “additiva di istituto”, cit., p. 3320; LORENZETTO, Elisa. Violazioni convenzionali, cit., p. 13.

${ }^{142}$ Proprio a questi fini, oltreché per le valutazioni di merito che l'accertamento dei presupposti della rescissione impone, quest'ultimo rimedio - come sopra ricordato - è stato sottratto dalla 1. 103 del 2017 al giudice di legittimità. 
CAPRIOLI, Francesco. Giudicato e illegalità della pena: riflessioni a margine di una recente sentenza della Corte costituzionale. In: BARGIS, Marta (a cura di). Studi in ricordo di Maria Gabriella Aimonetto. Milano: Giuffré, 2013, p. 263.

CAPRIOLI, Francesco. Abuso del diritto di difesa e nullità inoffensive. Cassazione penale, 2012, p. 2444.

CARNEVALE, Stefania. L'inidoneità dei rimedi individuati dalla giurisprudenza. Diritto penale e processo, 2007, p. 1234.

CARVELLI, Chiara. Rescissione del giudicato e reformatio in peius. Diritto penale e processo, 2014, p. 1039.

CASIRAGHI, Roberta. La rescissione del giudicato: molte questioni interpretative sul tappeto. Rivista italiana di diritto e procedura penale, 2018, p. 207.

CASSIBBA, Fabio. Brevi riflessioni sull'inutilizzabilità delle dichiarazioni estorte con tortura ai sensi del nuovo art. 191 comma 2-bis, in <https://penalecontemporaneo.it>, 2018, n. 4, p. 109. Accesso: 13 agosto 2018.

CAVALLARO, Tiziana. La revisione contra reum del giudicato penale tra realtà e prospettive di riforma. Diritto penale e processo, 2010, p. 1107.

CONTI, Giovanni. Il processo in absentia: le ricadute sul giudicato, in <http:// www.penalecontemporaneo.it>, 2 marzo 2015. Accesso: 14 agosto 2018.

CORVI, Paola, La revisione in peius. In CORVI, Paola (a cura di). Le impugnazioni straordinarie. Torino: Giappichelli, 2016, p. 95.

COZZI, Alessia Ottavia. L'impatto delle sentenze della Corte di Strasburgo sulle situazioni giuridiche interne definite da sentenze passate in giudicato: la configurabilità di un obbligo di riapertura o revisione del processo. In: SPITALERI, Fabio (a cura di). L'incidenza del diritto comunitario e della CEDU sugli atti nazionali definitivi. Milano: Giuffré, 2009, p. 159.

DE SALVIA, Michele. L'obbligo degli Stati di conformarsi alle decisioni della Corte europea e del Comitato dei ministri del Consiglio d'Europa. In: BALSAMO, Antonio; KOSTORIS, Roberto (a cura di). Giurisprudenza europea e processo penale italiano. Torino: Giappichelli, 2008, p. 67.

DIDDI, Alessandro. Novità in materia di impugnazioni e di restitutio in integrum. In: VIGONI, Daniela (a cura di), Il giudizio in assenza dell'imputato. Torino: Giappichelli, 2014, p. 209.

DI PAOLO, Gabriella. I presupposti della rescissione del giudicato. In: CORVI, Paola (a cura di). Le impugnazioni straordinarie. Torino: Giappichelli, 2016, p. 201. 
FALATO, Fabiana. La relatività del giudicato processuale. Napoli: Editoriale Scientifica, 2016.

FERRUA, Paolo. La prova nel processo penale, Torino: Giappichelli, 2015.

FERRUA, Paolo. Il 'giusto processo'. Bologna: Zanichelli, 3ª ed., 2012.

GALANTINI, Novella. La riassunzione della prova dichiarativa in appello: note a margine di Sezioni unite Troise. In: <http://www.penalecontemporaneo.it>, 17 aprile 2018. Accesso: 14 agosto 2018.

GERACI, Rosa Maria. Sentenze della Corte E.D.U. e revisione del processo penale. Roma: Dike, 2012.

GERACI, Rosa Maria. L'impugnativa straordinaria per la violazione della Cedu accertata a Strasburgo. Le ipotesi, le procedure, gli effetti. In CORVI, Paola (a cura di). Le impugnazioni straordinarie. Torino: Giappichelli, 2016, p. 69.

GIALUZ, Mitja. Una sentenza "additiva di istituto": la Corte costituzionale crea la "revisione europea”. Cassazione penale, 2011, p. 3308.

IACOVIELLO, Francesco Maria. Il quarto grado di giurisdizione: la Corte europea dei diritti dell'uomo. Cassazione penale, 2011, p. 794.

KOSTORIS, Roberto. Diritto europeo e giustizia penale. In: KOSTORIS, Roberto (a cura di.). Manuale di procedura penale europea. Torino: Giappichelli, $3^{\text {a }}$ ed., 2017, p. 1.

KOSTORIS, Roberto. La revisione del giudicato iniquo e i rapporti tra violazioni convenzionali e regole interne. Legislazione penale, 2011, p. 473.

LAVARINI, Barbara. I rimedi post iudicatum alla violazione dei canoni europei. In GAITO, Alfredo (a cura di). I princìpi europei del processo penale. Roma: Dike, 2016, p. 87.

LAVARINI, Barbara. Giudicato penale, ed esecuzione delle sentenze della Corte europea dei diritti dell'uomo. In: GAMBINI, Rosanna; SALVADORI, Margherita (a cura di). Convenzione europea sui diritti dell'uomo: processo penale e garanzie. Napoli: ESI, 2009, p. 129.

LOGLI, Andrea. La riapertura del processo a seguito della sentenza Cedu. Questioni interpretative sul nuovo caso di "revisione europea”. Cassazione penale, 2012, p. 933.

LORENZETTO, Elisa. Soltanto in Cassazione o anche in appello, questo è il dilemma, in <http://www.lalegislazionepenale.eu/>, 7 dicembre 2017. Accesso: 14 agosto 2018 . 
LORENZETTO, Elisa. Violazioni convenzionali e tutela post iudicatum dei diritti umani, in <http://www.lalegislazionepenale.eu>, 25 novembre 2016. Accesso: 13 agosto 2018.

MANTOVANI, Giulia. La sentenza n. 129 del 2008 e la riparazione delle violazioni dell'art. 6 CEDU. Giurisprudenza costituzionale, 2008, p. 2679.

MARCHETTI, Maria Riccarda. Commento all'art. 4, legge 9 aprile 1990, n. 88 di ratifica ed esecuzione del Protocollo n. 7 alla Convenzione europea per la salvaguardia dei diritti dell'uomo e delle libertà fondamentali. Legislazione penale, 1991, p. 227.

MAZZA, Oliviero. Cedu e diritto interno. In: GAITO, Alfredo. I princìpi europei del processo penale. Roma: Dike, 2016, p. 3.

MAZZA, Oliviero. La procedura penale. In VIGANÒ, Francesco; MAZZA, Oliviero. Europa e giustizia penale. Diritto penale e processo. Speciali, Assago, 2011, p. 33.

MAZZA, Oliviero. L'esecuzione può attendere: il caso Dorigo e la condanna ineseguibile per accertata violazione della CEDU. Giurisprudenza italiana, 2007, p. 2637.

NEGRI, Daniele. Splendori e miserie della legalità processuale. Archivio penale, 2017, n. 2, p. 421. https://doi.org/10.12871/9788674101944

NEGRI, Daniele. I confini della legalità processuale. Diritto penale e processo, 2007, p. 1229

PADULA, Carlo. La Corte Edu e i giudici comuni nella prospettiva della recente giurisprudenza costituzionale, in <http://www.giurcost.org/studi/ >, 2016, n. 11, p. 317 . Accesso: 13 agosto 2018.

PARLATO, Lucia. Revisione del processo iniquo: la Corte costituzionale "getta il cuore oltre l'ostacolo". Diritto penale e processo, 2011, p. 839.

PIRRONE, Pasquale. Sub art. 46. In: BARTOLE, Sergio; DE SENA, Pasquale; ZAGREBELSKY, Vladimiro (a cura di). Commentario breve alla CEDU. Padova: Cedam, 2012, p. 744.

QUATTROCOLO, Serena. Assenza e irreperibilità dell'imputato. Enciclopedia del diritto, Annali, IX. Milano: Giuffrè, 2016, p. 29.

QUATTROCOLO, Serena. Il contumace cede la scena processuale all'assente, mentre l'irreperibile l'abbandona. Diritto penale contemporaneo, Riv. trim, 2014, n. 2, p. 97 (disponibile in <http://www.penalecontemporaneo.it>. Accesso: 14 agosto 2018). 
QUATTROCOLO, Serena. Violazioni di convenzioni internazionali e giudicato penale interno. Rivista di diritto processuale, 2012, p. 647.

SCALFATI, Adolfo. L'esame sul merito nel giudizio preliminare di revisione. Padova: Cedam, 1995.

SCIARABBA, Vincenzo. Il giudicato e la Cedu. Padova: Cedam, 2012.

SPAGNOLO, Paola. La rinnovata fisionomia della rescissione del giudicato. In: BARGIS, Marta; BELLUTA, Hervè (a cura di). La riforma delle impugnazioni tra carenze sistematiche e incertezze applicative, Torino: Giappichelli, 2017, p. 141.

SPAGNOLO, Paola. Un istituto nuovo e uno vecchio: la rescissione del giudicato e la restituzione nel termine. Legislazione penale, 2014, n. 4, p. 636.

TONINI, Paolo; CONTI, Carlotta. Il tramonto della contumacia, l'alba radiosa della sospensione e le nubi dell'assenza “consapevole”. Diritto penale e processo, 2014, p. 509.

TROISI, Paolo. Flessibilità del giudicato e tutela dei diritti fondamentali, in <http:// www.penalecontemporaneo.it>, 2 aprile 2015. Accesso: 13 agosto 2018.

UBERTIS, Giulio. La revisione successiva a condanne della Corte di Strasburgo. Giurisprudenza costituzionale, 2011, p. 1542.

UBERTIS, Giulio. Corte europea dei diritti dell'uomo e processo equo: riflessi sul processo penale italiano. Rivista di diritto processuale, 2009, p. 33.

UBERTIS, Giulio. Conformarsi alle condanne europee per violazione dell'equità processuale: doveroso e già possibile. Corriere del merito, 2007, p. 599.

VOENA, Giovanni Paolo. Atti. In: CONSO, Giovanni; GREVI, Vittorio; BARGIS, Marta. Compendio di procedura penale. Padova: Cedam, 8 ed., 2016, p. 173.

ZACCHÉ, Francesco. Cassazione e iura novit curia nel caso Drassich. Diritto penale e processo, 2009, p. 781. 


\section{Informações adicionais e declarações dos autores (integridade científica)}

Declaração de conflito de interesses (conflict of interest declaration): a autora confirma que não há conflitos de interesse na realização das pesquisas expostas e na redação deste artigo.

Declaração de autoria e especificação das contribuições (declaration of authorship): todas e somente as pessoas que atendem os requisitos de autoria deste artigo estão listadas como autores.

Declaração de ineditismo e originalidade (declaration of originality): a autora assegura que o texto aqui publicado não foi divulgado anteriormente em outro meio e que futura republicação somente se realizará com a indicação expressa da referência desta publicação original; também atesta que não há plágio de terceiros ou autoplágio.

Dados do processo editorial

(http://www.ibraspp.com.br/revista/index.php/RBDPP/about/editorialPolicies)

- Recebido em: 18.07.2018

- Controle preliminar e verificação de plágio: 19.07.2018

- Avaliação 1: 29.07.2018

- Avaliação 2: 07.08.2018

- Decisão editorial preliminar: 19.07.2018

- Retorno rodada de correções 1: 04.09.2018

- Decisão editorial final: 22.09.2018

\section{Equipe editorial envolvida}

- Editor-chefe: 1 (VGV)

- Editores-associados: 2 (BC FC)

- Revisores: 2 
COMO CITAR ESTE ARTIGO:

LAVARINI, Barbara. Violazione delle garanzie "processuali" della CEDU e rimedi post-iudicatum. Revista Brasileira de Direito Processual Penal, Porto Alegre, vol. 4, n. 3, p. 1003-1052, set./dez. 2018. https://doi.org/10.22197/rbdpp.v4i3.184 